\title{
Systematic Modeling of Discrete-Continuous Optimization Models through Generalized Disjunctive Programming
}

\author{
Ignacio E. Grossmann ${ }^{1}$ and Francisco Trespalacios \\ Center for Advanced Process Decision-making \\ Department of Chemical Engineering \\ Carnegie Mellon University \\ Pittsburgh, PA 15213
}

\begin{abstract}
This article is dedicated to the memory of Professor Neil Amundsen, who pioneered the application of mathematical modeling and analysis in chemical engineering.
\end{abstract}

\begin{abstract}
Discrete-continuous optimization problems in process systems engineering are commonly modeled in algebraic form as mixed-integer linear or nonlinear programming models. Since these models can often be formulated in different ways, there is a need for a systematic modeling framework that provides a fundamental understanding on the nature of these models, particularly their continuous relaxations. This paper describes a modeling framework, Generalized Disjunctive Programming (GDP), which represents problems in terms of Boolean and continuous variables, allowing the representation of constraints as algebraic equations, disjunctions and logic propositions. We provide an overview of major research results that have emerged in this area. Basic concepts are emphasized as well as major classes of formulations that can be derived. These are illustrated with a number of examples in the area of process systems engineering. As will be shown, GDP provides a structured way for systematically deriving mixed-integer optimization models that exhibit strong continuous relaxations.
\end{abstract}

\section{Introduction}

Mixed-integer optimization provides a powerful framework for the mathematical modeling of many optimization problems that involve discrete and continuous variables. Over the last few years there has been a pronounced increase in the development of Mixed-integer Linear/ Nonlinear Programming (MILP/MINLP) models in Process Systems Engineering ${ }^{\text {[10][12][21][23][29]. }}$ Synthesis models can be formulated as MILP for high level targeting, and as MINLP for more detailed superstructure optimization ${ }^{[11]}$. For planning and scheduling, the majority tends to be MILP models ${ }^{[6][9][24][28]}$ although gradually there is also an increasing trend to MINLP, especially as process models are incorporated ${ }^{[17]}$. MILP/MINLP models, however, are based on algebraic formulations which are not unique. Although there has been significant progress in software for solving mixed-integer problems, especially MILP, how one formulates a model can have a major impact in the performance and capability to find a solution. Therefore, deriving "good" formulations or finding potential improvements in existing models is commonly regarded as an art and strongly depends on the modeler skills.

\footnotetext{
${ }^{1}$ Author to whom correspondence should be addressed. E-mail: grossmann@cmu.edu
} 
Generalized Disjunctive Programming (GDP) originated with the goal of facilitating the modeling of discrete/continuous optimization problems through the use of higher level logic constructs ${ }^{[19][32]}$. This approach involves algebraic equations, disjunctions and logic propositions in the formulation of a model. Unlike direct MINLP formulations, this higher level modeling framework makes the formulation process more intuitive and systematic, while retaining in the model the underlying logic structure of the problem. Although there are some special techniques to solve this type of problems, such as Disjunctive Branch and Bound ${ }^{[26]}$ and Logic Based Outer Approximation $^{[40]}$, GDPs are normally directly reformulated as MILP/MINLP ${ }^{[25][30]}$ to exploit the developments in these solvers (A review on MINLP methods is provided by Grossmann ${ }^{[13]}$ ) .

The focus on this paper is on the systematic formulation of MILP/MINLP models using GDP as a theoretical framework. In the first section we introduce the concept of GDP and provide some chemical engineering illustrations. We next examine the process for transforming GDP to MILP/MINLP models, describing alternative reformulations and application of logic tools to improve the formulations. Finally, we illustrate the application of these concepts on several problems.

\section{Generalized Disjunctive Programming}

An alternative approach for representing discrete/continuous optimization problems is by modeling them using algebraic equations, disjunctions and logic propositions ${ }^{\text {[3][19][20][25][32][40] }}$. Such a model is known as Generalized Disjunctive Programming ${ }^{[16][32]}$, the main focus of this paper, which can be regarded as a generalization of disjunctive programming developed by Balas $^{[1]}$. Process Design ${ }^{[12][40][45]}$ and Planning and Scheduling ${ }^{[6][17]}$ are some of the areas where GDP formulations have shown to be successful.

\subsection{Motivation}

In order to illustrate that the way we formulate MILP/MINLP models can have a major impact in time and capability to find a solution, consider the following simple example. A company has to decide whether to produce either product $A$ or product $B$, in order to maximize its profit. The profit of product $A$ is 3 , and the profit of product $B$ is 2 . The limit on production of $A$ is 4 , and the limit in production of $\mathrm{B}$ is 5 . There are at least three different formulations for this problem (P1a), (P1b) and (P1c) that are given below.

(P1a):

$$
\begin{array}{ll}
\max z=3 A+2 B \\
\text { s.t. } & A * y_{2}=0 \\
& B * y_{1}=0 \\
& y_{1} * y_{2}=0 \\
& 0 \leq A \leq 4 \\
& 0 \leq B \leq 5 \\
& y_{1}+y_{2}=1 \\
& 0 \leq y_{1}, y_{2} \leq 1 \\
& A, B, y_{1}, y_{2} \in \mathbb{R}
\end{array}
$$

(P1b):

$$
\max z=3 A+2 B
$$

$$
\begin{array}{ll}
\text { s.t. } & A \leq 10-10 * y_{2} \\
& B \leq 10-10 * y_{1}
\end{array}
$$$$
0 \leq A \leq 4
$$$$
0 \leq B \leq 5
$$$$
y_{1}+y_{2}=1
$$$$
A, B \in \mathbb{R}
$$$$
y_{1}, y_{2} \in\{0,1\}
$$

(P1c):

$\max z=3 A+2 B$

$$
\begin{array}{ll}
\text { s.t. } & 0 \leq A \leq 4 * y_{1} \\
& 0 \leq B \leq 5 * y_{2}
\end{array}
$$

The basic idea in (P1a), (P1b) and (P1c) is to formulate the problem as an optimization problem in which the $0-1$ variables $y_{1}, y_{2}$ are needed to determine whether $\mathrm{A}$ or $\mathrm{B}$ are produced, 
respectively. The three formulations are valid representations of the problem, while (P1a) corresponds to a nonlinear program (NLP) due to the nonlinear functions involved, both (P1b) and (P1c) correspond to two alternative linear MILP models. Note that one can only set either $y_{1}$ or $y_{2}$ equal to 1 . When $y_{1}=1$ and $y_{2}=0$ then $B=0$ and $0 \leq A \leq 4$; when $y_{1}=0$ and $y_{2}=1$ then $A=0$ and $0 \leq B \leq 5$. Since all three formulations are valid MILP/NLP, they have the same optimal solution $\mathrm{z}_{\mathrm{NLP}}=\mathrm{z}_{\mathrm{MILP}}=12$. However, the solution efficiency is strongly linked to the type of model. (P1a) is nonlinear and nonconvex, which requires a global optimization algorithm and is therefore expected to be considerably slower than the other two small MILP formulations. In the MILPs, the difference between $\mathrm{z}_{\mathrm{MILP}}$ and the value of the optimal solution to the continuous LP relaxation $\mathrm{z}_{\mathrm{LP}}$, in which $y_{1}$ and $y_{2}$ are treated as continuous variables between 0 and 1 , is known as the relaxation gap. The relaxed solution $\mathrm{z}_{\mathrm{LP}}$ of (P1b) and (P1c) is 22 and 12 respectively. Since the relaxation gap in (P1b) is 10 while the gap in (P1c) is zero, the time to solve (P1c) is expected to be much faster than the other two formulations. Figure 1 illustrates the feasible region for $\mathrm{A}$ and $\mathrm{B}$, in the continuous relaxation of the formulations.

(P1a)

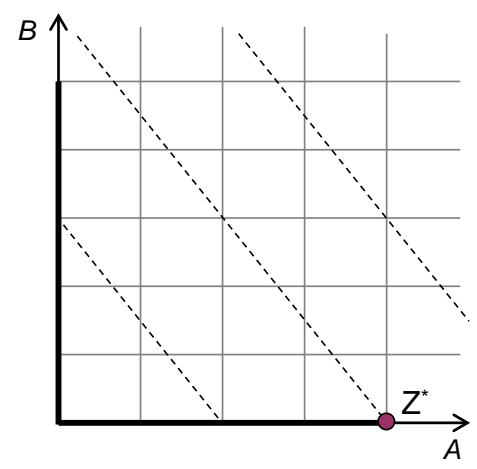

(P1b)

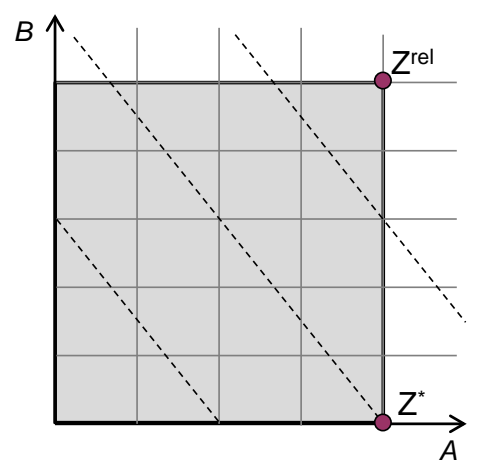

(P1c)

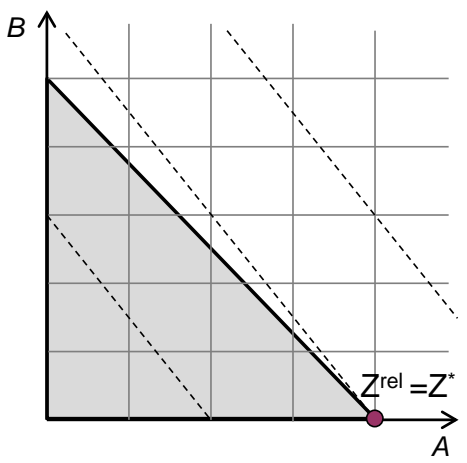

Figure 1. Feasible region of continuous relaxation of (P1a), (P1b) and (P1c) projected in A and $B$

Not only are there several ways in which to formulate MILP/MINLP problems, but also the transformation of a conditional argument into a constraint is not always obvious. Consider the following process synthesis requirement ${ }^{[4]}$ : (P2) "If the absorber to recover the product $\left(y_{a}\right)$ or the membrane separator $\left(y_{m}\right)$ is selected, then do not use cryogenic separation $\left(y_{c}\right)$ ". This logic condition can be modeled with any of the following three constraint(s), in which $y_{a}, y_{m}$ and $y_{c}$ are $0-1$ variables:

(P2a) $y_{a}+y_{m}+2 y_{c} \leq 2$

(P2b) $y_{a}+y_{c} \leq 1$

$y_{m}+y_{c} \leq 1$

(P2c) $y_{c}+y_{a}+y_{m}-y_{a} * y_{m} \leq 1$

While the three constraints above are all valid representations of the logic condition, with the first two being linear and the last nonlinear, the modeling of logic constraints can be made more systematic by using logic propositions in which the $0-1$ variables $y$ are replaced by Boolean variables $Y$. Following the previous example, the corresponding logic proposition is If $\left(Y_{a}\right.$ or $\left.Y_{m}\right)$ then $\left(\right.$ not $\left.Y_{c}\right)$. Or in standard Boolean logic: 
(P2d) $Y_{a} \vee Y_{m} \Rightarrow\left(\neg Y_{c}\right)$

As will be seen later, (P2d) can be systematically transformed into linear inequalities with $0-1$ variables, yielding constraints (P2b), which in fact provide a "tighter" formulation than (P2a) when relaxing the integrality constraints (i.e. considering the $0-1$ variables as continuous variables between 0 and 1 ).

The following notation (logic operators) will be used throughout the paper:

$\checkmark$ means OR (inclusive, i.e. A or B or both)

$\wedge$ means AND

$\neg$ means NOT (negation or complement)

$\Longrightarrow$ means IF...THEN... (implication)

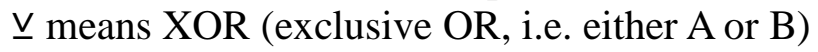

$\Leftrightarrow$ means IF AND ONLY IF

As shown in the next section, GDP is a higher-level representation that allows the use of logic arguments in optimization problems like in the above example. This approach not only facilitates the development of the models by making the formulation process more systematic and intuitive, but it also keeps in the model the underlying logic structure of the problem that can be exploited to find the solution more efficiently.

\subsection{GDP Formulation}

The general structure of a GDP can be represented as follows ${ }^{2}$ :

(GDP):

$$
\begin{array}{ll} 
& \min z=f(x) \\
\text { s.t. } & g(x) \leq 0 \\
& \underline{v}_{i \in D_{k}}\left[\begin{array}{c}
Y_{k i} \\
r_{k i}(x) \leq 0
\end{array}\right] \quad k \in K \\
& \Omega(Y)=\text { True } \\
& x \in \mathbb{R}^{n} \\
& Y_{k i} \in\{\text { True, False }\} \quad k \in K, i \in D_{k}
\end{array}
$$

As shown in (GDP), the objective is a function of the continuous variables $x$. It contains the global constraints $g(x) \leq 0$ that must hold true regardless of the discrete decisions. The logic in the continuous space is represented by a set of disjunctions $k \in K$, each of which contains $i \in D_{k}$ terms, linked by an XOR operator ( $\underline{\mathrm{v}}$ ). Each term of the disjunction has a Boolean variable $Y_{k i}$ and an associated set of inequalities $r_{k i}(x) \leq 0$. For an active term in a disjunction $\left(Y_{k i}=\right.$ True), the corresponding inequalities are enforced. When the term is not active $\left(Y_{k i}=F a l s e\right)$, the

${ }^{2}$ This is a slightly more general form than the one originally presented in [32] 
corresponding constraints are ignored. The symbolic equation $\Omega(Y)=$ True represents the set of logic propositions that relates the Boolean variables. Note that GDP is a more structured alternative for modeling discrete-continuous optimization problems compared to mixed-integer programming, which is represented as:

\section{(MINLP):}

$$
\begin{array}{ll} 
& \min Z=f(x, y) \\
\text { s.t. } & g(x, y) \leq 0 \\
& x \in \mathbb{R}^{n}, y \in\{0,1\}^{m}
\end{array}
$$

where $f(x, y)$ is the objective function, $g(x, y) \leq 0$ are inequality constraints, and $y$ are the $0-1$ variables, the counterpart to the Boolean variables $Y$. It should be noted that since (MINLP) is expressed through algebraic equations, the modeler has to directly express the logic in the format of $f(x, y)$ and $g(x, y)$, while in (GDP) the disjunctions capture the logic in continuous form and the logic propositions $\Omega(Y)$ capture the logic in the Boolean space.

While model (GDP) is quite general, in the Appendix we present the reformulation for the case of embedded disjunctions and inclusive or, in which the models are eventually transformed to the form of model (GDP). Note that in both general forms (GDP) and (MINLP), we only represent inequalities, since any equality can be considered as a set of inequalities (i.e. $h(x, y)=0$ can be expressed as the two inequality constraints $h(x, y) \leq 0$ and $-h(x, y) \leq 0)$.

\subsection{GDP in Process Systems Engineering}

Decision making is a major element in Process Systems Engineering (PSE), making GDP a very useful framework for modeling PSE optimization problems. Particularly in process synthesis, the decisions are normally associated as to whether certain equipment should be included or not in a process flowsheet. If the equipment is selected, then the mass and energy balance, physical and chemical equilibrium (if any), and cost constraints need to be satisfied. If it is not selected, then all the equations can be ignored. Therefore, in PSE GDP problems will normally take the following form ${ }^{[32]}$ :

\section{(GDP1):}

$$
\min z=\hat{f}(x)+\sum_{k \in K} c_{k}
$$

s.t. $g(x) \leq 0$

$$
\begin{aligned}
& \underline{\mathrm{v}}_{i \in D_{k}}\left[\begin{array}{c}
Y_{k i} \\
\hat{r}_{k i}(x) \leq 0 \\
c_{k}=\gamma_{k i}
\end{array}\right] \quad k \in K \\
& \Omega(Y)=\text { True } \\
& x \in \mathbb{R}^{n}, c_{k} \in \mathbb{R}^{1}, Y_{i k} \in\{\text { True, False }\}, i \in D_{k}, k \in K
\end{aligned}
$$


Note that (GDP1) is a particular case of (GDP), where $f(x)=\hat{f}(x)+\sum_{k \in K} c_{k}$ and $r_{k i}(x) \leq 0$ is $\hat{r}_{k i}(x) \leq 0$ and $c_{k}=\gamma_{k i}$ in (GDP1), where $c_{k}$ represents the cost associated with enforcing a term in the disjunction. In process synthesis the continuous variables normally represent flows, temperatures and pressures of the stream in a process superstructure, while the Boolean variables represent the selection of equipment $i$ for performing a process task $k$. The objective is a function of the continuous variables and the cost associated with each of the disjunctions $k$. The global constraints $g(x) \leq 0$ normally represent mass and energy balances in the system. For an active term in a disjunction, $Y_{k i}=$ True, the cost $c_{k}=\gamma_{i k}$ and the constraints $\hat{r}_{k i}(x) \leq 0$ are normally associated with the investment cost, the energy and mass balance, and physical and chemical equilibrium for the particular equipment $i$ that is selected for the processing task $k$. The argument $\Omega(Y)=$ True generally represents the logic implications among the equipment in order to define a feasible topology for the process flowsheet. The logic constraints may be "hard" constraints like specification of choices, or redundant constraints in the sense that they may be implied by the mass balances at a process network. In the latter case the logic constraints can help to expedite the search for the optimum

(GDP1) provides a general and systematic framework for modeling process networks. Although it is a particular case of (GDP), it has some MILP/MINLP reformulation advantages that will be discussed in section 3.2 of this paper.

\subsection{Illustrative examples}

We illustrate the concept of the model (GDP) with four PSE examples: Synthesis of a process flowsheet, determination of number of trays in a distillation column, job shop scheduling, and design of a batch process.

\subsubsection{Process Synthesis}

Consider the optimization of a simple process superstructure shown in Figure 2 that produces a product $\mathrm{B}$ by consuming raw material $\mathrm{A}$. The variables $\mathrm{F}$ represent material flows. The problem is to determine the amount of product to produce $\left(\mathrm{F}_{8}\right)$ with a selling price $\mathrm{P}_{8}$, the amount of raw material to buy $\left(\mathrm{F}_{1}\right)$ with a cost $\mathrm{P}_{1}$ and the set of unit operations to use (i.e. HX1, R1, R2, DC1) with a cost $c_{k}$, in order to maximize the profit ${ }^{[33]}$.

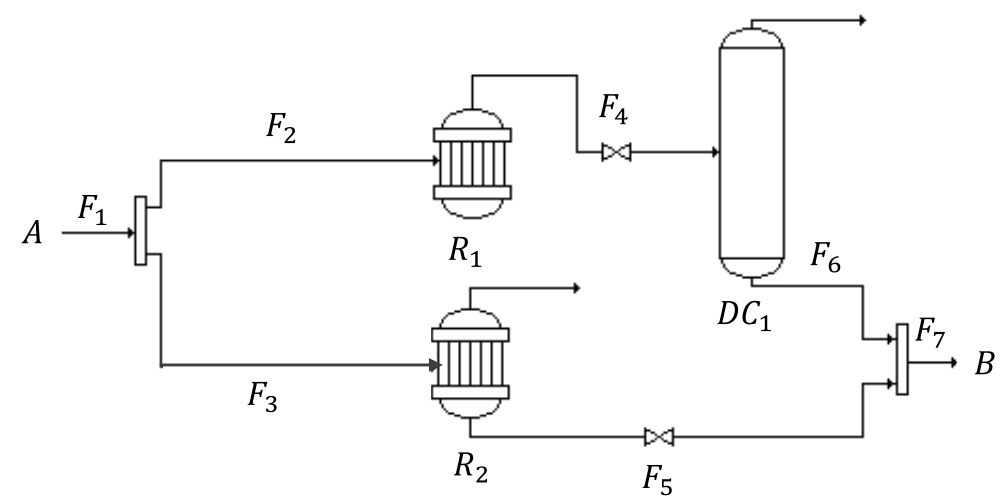

Figure 2. Process network example

The GDP that represents the problem can be formulated as model (GDP1) as follows: 
(P3):

$$
\max z=P_{7} F_{7}-P_{1} F_{1}-\sum_{k \in K} c_{k}
$$

s.t.

$$
\begin{aligned}
& F_{1}=F_{2}+F_{3} \\
& F_{7}=F_{5}+F_{6}
\end{aligned}
$$

$\left[\begin{array}{c}Y_{R 2} \\ F_{5}=\beta_{R 2} F_{3} \\ c_{R 2}=\gamma_{R 2}\end{array}\right] \underline{v}\left[\begin{array}{c}\neg Y_{R 2} \\ F_{3}=F_{5}=0 \\ c_{R 2}=0\end{array}\right]$

$$
\begin{aligned}
& {\left[\begin{array}{c}
Y_{R 1} \\
F_{4}=\beta_{R 1} F_{2} \\
c_{R 1}=\gamma_{R 1}
\end{array}\right] \underline{\mathrm{v}}\left[\begin{array}{c}
\neg Y_{R 1} \\
F_{2}=F_{4}=0 \\
c_{R 1}=0
\end{array}\right]} \\
& {\left[\begin{array}{c}
Y_{D C 1} \\
F_{6}=\beta_{D C 1} F_{4} \\
c_{D C 1}=\gamma_{D C 1}
\end{array}\right] \underline{\mathrm{v}}\left[\begin{array}{c}
\neg Y_{D C 1} \\
F_{4}=F_{6}=0 \\
c_{D C 1}=0
\end{array}\right]}
\end{aligned}
$$

$Y_{R 1} \Leftrightarrow Y_{D C 1}$

$F_{j} \geq 0$

$$
\begin{array}{ll}
F_{j} \geq 0 & j=1,7 \\
F_{j}, c_{k} \in \mathbb{R}, Y_{k} \in\{\text { True, False }\} & j=1,7
\end{array}
$$

In the above, equation (1) represents the objective function; (2) are the global constraints representing the mass balances around the splitter and mixer respectively; (3) are the disjunctions that represent the selection or not of the unit operation $k$, with their respective characteristic constraints and fixed costs; and (4) the logic propositions that enforce the selection of DC1 if and only if R1 is chosen, and HX1 if and only if R2 is chosen. For illustration purposes we have presented here a simple linear model. In the actual application to a process problem there would be hundreds or thousands of nonlinear equations in the GDP model.

\subsubsection{Distillation Column Design}

The optimal design of selecting the feed tray and the number of trays in a distillation column has remained a major challenge since the pioneering work by Sargent and Gaminibanadara ${ }^{\text {[36] }}$ reported in 1976. Viswanathan and Grossmann ${ }^{[43]}$ proposed an MINLP formulation involving a superstructure with variable reflux location as depicted in Figure 3. The idea is to consider a fixed feed tray and a fixed number of trays above and below the feed that represent an upper bound for the rectifying and stripping sections, respectively. The reflux is returned to any of the trays above the feed, and the reboil to any tray below the feed. A 0-1 variable $z r_{i}, z b_{i}$ is assigned to the reflux and reboil return to each tray $i$, respectively. The solution of the MINLP assigns only one binary variable $z r_{i}$ to one, for the return of the reflux into the selected tray, so that all the trays above the selected tray are not required in the optimal design. Similarly, there is only one binary variable $z b_{i}$ equal to one in the reboil return, and all the trays below this selected tray are not included in the optimal design. The main difficulty with this approach is that in the trays that are not selected (i.e. trays are inexistent and there is no mass transfer), vapor liquid equilibrium (VLE) equations still have to be satisfied ${ }^{[15][43]}$. 


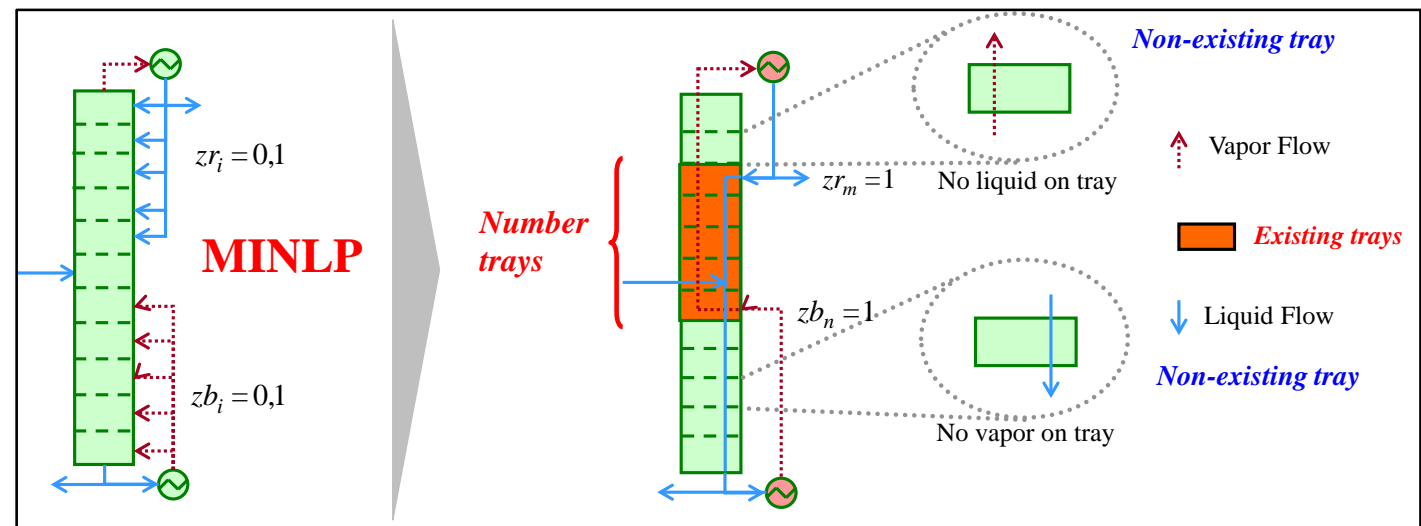

Discrete variables: Number of trays, feed tray location.

Continuous variables: reflux ratio, heat loads, exchanger areas, column diameter.

Figure 3. Variable reflux and reboil location with fixed feed tray

In contrast, Yeomans and Grossmann ${ }^{[45]}$ proposed a GDP model for the same problem. In this model the selection of the trays is represented with the Boolean variables $Y_{n}$. If a certain tray is selected $\left(Y_{n}=\right.$ True $)$, then the VLE equations have to be applied for that tray; if is not selected $\left(Y_{n}=\right.$ False), then there is no heat and mass transfer in the tray (e.g. simply a "by-pass"). In this way, this formulation overcomes the difficulties of satisfying VLE in trays that are not selected. Figure 4 shows a column representation for this approach.

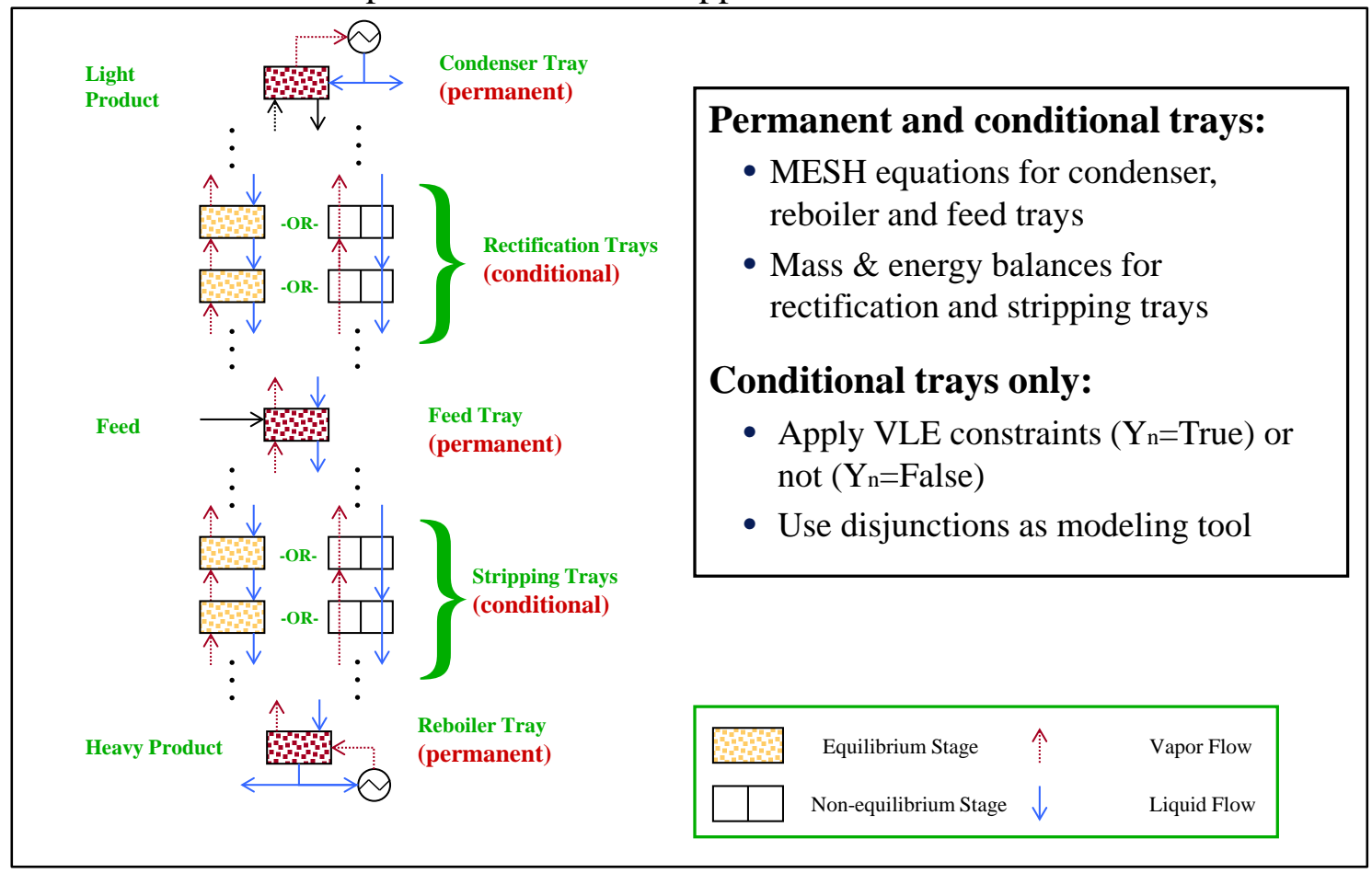

Figure 4. Structure of disjunctive model with permanent and conditional trays

Given the complexity of the formulation, we present the GDP model as shown in equation (P4), without explicitly including all the well-known equations. 
(P4):

Min Cost

s.t.

MESH equations for permanent trays

Mass / energy balances for conditional trays

$$
\begin{aligned}
& {\left[\begin{array}{c}
Y_{n} \\
f_{n, i}^{L}=f\left(T_{n}, P_{n}, x_{n, i}\right) \\
f_{n, i}^{V}=f\left(T_{n}, P_{n}, y_{n, i}\right) \\
f_{n, i}^{L}=f_{n, i}^{V} \\
T_{n}^{L}=T_{n}^{V} \\
L I Q_{n, i}^{V}=L_{n} x_{n, i} \\
V A P_{n, i}=V_{n} y_{n, i} \\
\operatorname{stg}_{n}=1
\end{array}\right] \underline{\mathrm{v}}\left[\begin{array}{c}
\neg Y_{n} \\
f_{n, i}^{L}=0 \\
f_{n, i}^{V}=0 \\
T_{n}^{L}=T_{n-1}^{L} \\
T_{n}^{V}=T_{n+1}^{V} \\
L_{n}=L_{n-1} \\
V_{n}=V_{n+1} \\
x_{n, i}=x_{n-1, i} \\
y_{n, i}=y_{n+1, i} \\
\text { stg }=0
\end{array}\right]} \\
& \text { ntray }=\sum s t g_{n} \\
& \Omega(Y)=\text { True }
\end{aligned}
$$

Note that if a tray is not selected $\left(\neg Y_{n}\right.$ ) the inlet and outlet flows, composition and temperatures of the liquid and vapor remain unchanged, while the fugacity is set to zero.

\subsubsection{Job shop scheduling}

Consider a job shop scheduling problem where a set of jobs $i \in I$ must be processed sequentially on a set of consecutive stages $j \in J$, with given processing times for each stage $\tau_{i j}$. All jobs can be sequenced on a subset of stages $j \in J(i)$. Furthermore, zero-wait transfer is assumed between stages, and the objective is to obtain a schedule that minimizes the makespan $m s$. A small example of such a problem is given in Table 1 , where it can be seen that job A requires only stages 1 and 3 , job B, stages 2 and 3 , and C stages 1 and 2 .

Table 1. Processing times $\left(\tau_{i j}\right)$ for small illustrative example.

\begin{tabular}{cccc}
\hline Jobs/ Stages & 1 & 2 & 3 \\
\hline A & 5 & 0 & 3 \\
B & 0 & 3 & 2 \\
C & 2 & 4 & 0 \\
\hline
\end{tabular}

By defining the Boolean variables $Y_{i k}^{1}$ and $Y_{i k}^{2}$ to indicate whether job $i$ is executed before job $k$ or job $k$ before job $i$, respectively, Raman and Grossmann ${ }^{[32]}$ proposed the following model: 
(P5):

$\min m s$

s.t.

$$
\begin{aligned}
& m s \geq t_{i}+\sum_{j \in J(i)} \tau_{i j} \quad \forall i \in I \\
& {\left[\begin{array}{c}
Y_{i k}^{1} \\
t_{i}+\sum_{\substack{m \in J(i) \\
m \leq j}} \tau_{i m} \leq t_{k}+\sum_{\substack{m \in J(k) \\
m<j}} \tau_{k m}
\end{array}\right] \underline{\mathrm{v}}} \\
& {\left[\begin{array}{c}
Y_{i k}^{2} \underset{m<j}{m \leq j} \\
t_{k}+\sum_{\substack{m \in J(k) \\
m \leq j}} \tau_{k t_{i}}+\sum_{m \in J(i)} \tau_{i m}
\end{array}\right] \quad \forall j \in C_{i k} \forall i, k \in I, i<k} \\
& m s, t_{i} \in \mathbb{R}, \quad Y_{i k}^{1}, Y_{i k}^{2} \in\{\text { True, False }\} \quad \forall i, k \in I, i<k
\end{aligned}
$$

in which $t_{i}$ is the start time of job $i$. Equations (P5.1) and (P5.2) correspond to the objective function and aim to minimize the makespan $m s$. The disjunction in (P5.3) ensures that no clash between jobs occurs at any stage at the same time, where for each pair of jobs $i, k$, the stages with potential clashes are $C_{i k}=\{J(i) \cap J(k)\}$. More specifically, the first term in each disjunction states that at a given stage $j$ the completion time of job $i$ is less or equal to the start of job $k$, and vice versa in the second term of each disjunction. Note that this requires the second job to have its processing times summed up to one stage less $(m<j)$ than the first job $(m \leq j)$, to represent start and completion times respectively. The optimal schedule for the small example in Table 1, which has a minimum makespan of 11 hours, is shown in Figure 5.

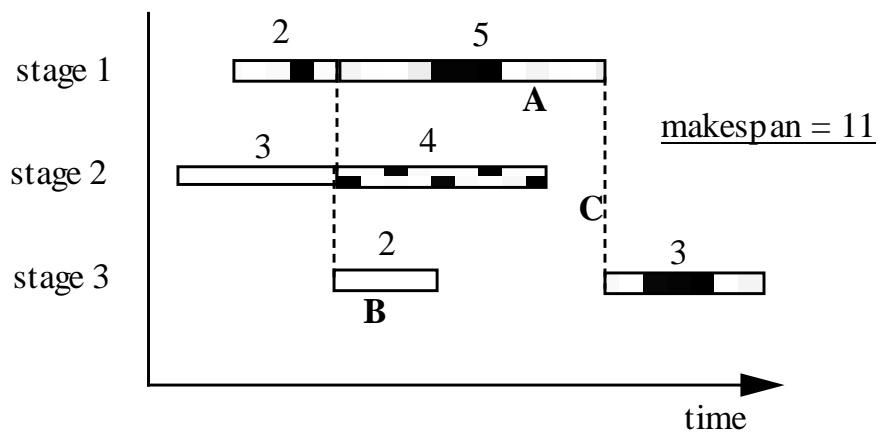

Figure 5. Optimal Schedule for Illustrative Example

\subsubsection{Synthesis of Multiproduct Batch Plant}

The simultaneous synthesis, sizing, and scheduling of a flowshop multiproduct batch plants can be stated as follows ${ }^{[5]}$. It is desired to design a batch plant to produce different products $(i=$ $\left.1, \ldots, N_{p}\right)$ in a sequence of stages. The manufacturing of all of these products requires that they undergo a sequence of processing tasks $(t=1, \ldots, T)$ in exactly the same order to obtain the final products. There are different types of units available $(j=1, \ldots, M)$. Production requirements $Q_{i}$ of 
the different products are specified over a given time horizon $H$. Processing times $p t_{i t j}$ and size factors $S_{i t}$ (volume required per unit batch size) are given for each product $i$ at the processing task $t$. To simplify the problem, cleanup times are assumed to be negligible. Each of the units $j$ is capable of performing a corresponding task or a subset of the $T$ tasks. The problem then consists in determining the structure of the plant by deciding which tasks should be assigned to which unit, the number of parallel equipment for each of these units, and their corresponding sizes. Also, a production schedule must be determined that will ensure that the plant will be able to meet the production requirements over the given horizon time. Since the plant to be synthesized is a flowshop network, the tasks to be merged in any processing unit need to be adjacent. In addition, the cost of each unit is assumed to be given by an equation of the form $C_{j}=\gamma_{j}+\alpha_{j} V_{j}^{\beta}$, where $\gamma$ is a fixed cost charge and $\alpha$ and $\beta$ are cost parameters for the unit size $V$. For simplicity, the sizes are assumed to be continuous within specified lower and upper bounds $V^{L}$ and $V^{U}$. Finally, the scheduling policies of unlimited intermediate storage (UIS) and zero wait (ZW) are considered for both types of campaigns, single and mixed product campaigns (SPCs and MPCs). The GDP formulation for the superstructure in Figure 6 is given by (P6):

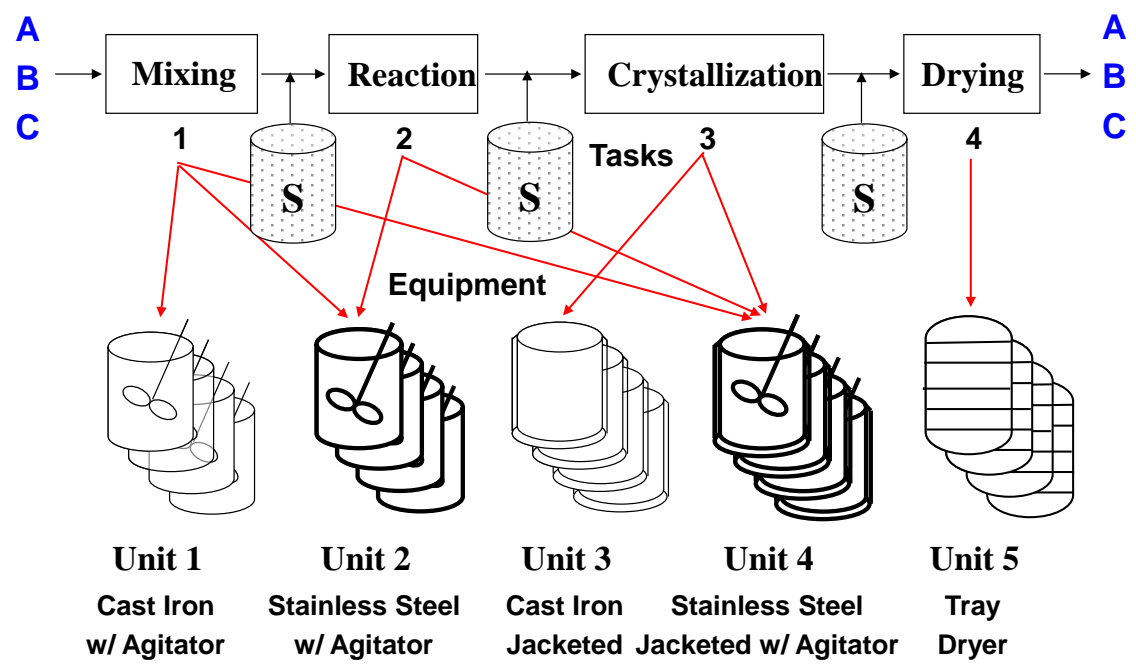

Figure 6. Illustrative Superstructure for Multiproduct Batch Plant Synthesis

(P6):

$$
\begin{aligned}
& \min C O S T=\sum_{j=1}^{M} N_{j}^{E Q} C_{j}+\sum_{j} C S_{j} \\
& \text { s.t. } \quad V_{t}^{T} \geq B_{i} S_{i t} \quad i=1, \ldots, N_{P} ; t=1, \ldots, T \\
& p t_{i j}=\sum_{t \in T_{j}} p t y_{i t j} \quad i=1, \ldots, N_{P} ; j=1, \ldots, M \\
& n_{i} B_{i} \geq Q_{i} \quad i=1, \ldots, N_{P} \\
& \sum_{i=1}^{N_{p}} n_{i} T_{L i} \leq H
\end{aligned}
$$


$\underset{v}{\underline{v}}\left[\begin{array}{c}Y_{t j} \\ V_{j} \geq V_{t}^{T} \\ p t y_{i t j}=p t_{i t}^{T} \\ p t y_{i t j^{\prime}}=0, j^{\prime} \neq j\end{array}\right] t \in T$

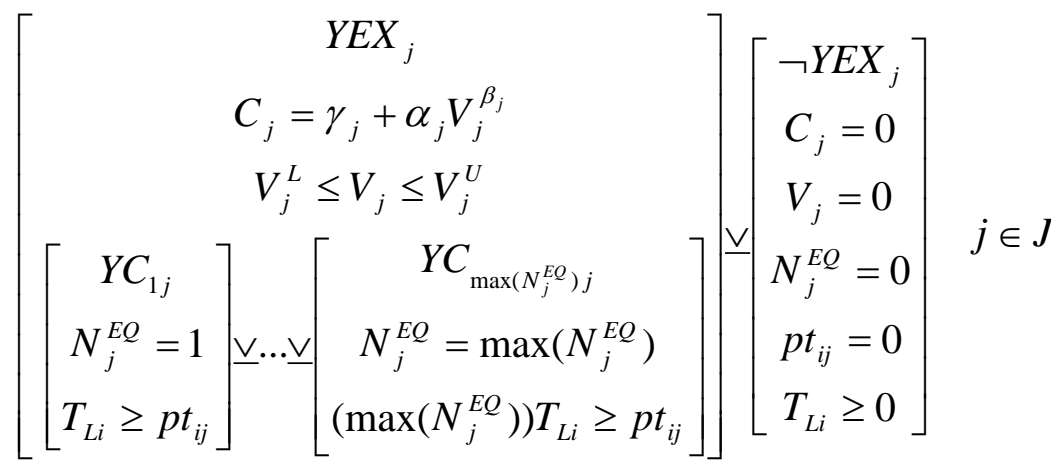

$\left[\begin{array}{c}Y S_{j} \\ -\phi \leq B_{i j}-B_{i j^{\prime}} \leq \phi \\ V S T_{j} \geq S^{\prime}{ }_{i j} B_{i j} N E Q_{j} \\ V S T_{j} \geq S_{i j}^{\prime} B_{i j^{\prime}} N E Q_{j^{\prime}} \\ V S T_{j}^{L B} \leq V S T_{j} \leq V S T_{j}^{U B} \\ C S_{j}=\gamma S_{j}+\alpha S_{j} V S T_{j}^{\beta S}\end{array}\right] \underline{\vee}\left[\begin{array}{c}\neg Y S_{j} \\ B_{i j}=B_{i j^{\prime}} \\ V S T_{j}=0 \\ C S_{j}=0\end{array}\right] j \in J$

$Y E X_{1} \Leftrightarrow Y_{11}$

$\mathrm{YEX}_{2} \Leftrightarrow Y_{12} \vee Y_{22}$

$Y E X_{3} \Leftrightarrow Y_{33}$

$Y E X_{4} \Leftrightarrow Y_{14} \vee Y_{24} \vee Y_{34}$

$Y E X_{5} \Leftrightarrow Y_{45}$

$W_{04} \underline{\vee} W_{14} \underline{\vee} W_{24} \underline{\vee} W_{34}$

$W_{04} \Leftrightarrow \neg Y_{14} \wedge \neg Y_{24} \wedge \neg Y_{34}$

$W_{14} \Leftrightarrow\left(Y_{14} \wedge \neg Y_{24} \wedge \neg Y_{34}\right) \underline{\vee}\left(\neg Y_{14} \wedge Y_{24} \wedge \neg Y_{34}\right) \underline{\vee}\left(\neg Y_{14} \wedge \neg Y_{24} \wedge Y_{34}\right)$

$W_{24} \Leftrightarrow\left(Y_{14} \wedge Y_{24} \wedge \neg Y_{34}\right) \underline{\vee}\left(\neg Y_{14} \wedge Y_{24} \wedge Y_{34}\right)$

$W_{34} \Leftrightarrow Y_{14} \wedge Y_{24} \wedge Y_{34}$

$0 \leq C_{j}, V_{j}, V_{t}^{T}, n_{i}, B_{i}, T_{L i}, p t_{i j}, N_{j}^{E Q}, p t y_{i t j} ; Y E X_{t j}, Y_{t j}, Y C_{c j}, Y S_{j}, W_{l j} \in\{$ true, false $\}$

In the above, the global constraints define equations for sizing, satisfying demands for each product and completing the production time within the specified time horizon $H$. The first set of disjunctions refers to the assignment of a task $t$ to an equipment $j$. The second disjunction corresponds to the selection of equipment $j$, which within its term has an embedded disjunction 
for selecting the number of parallel units (See Appendix 1 for embedded disjunctions). The third disjunction is for deciding whether to install a storage tank after unit $j$. The logic constraints define the feasible assignments of tasks $\left(Y_{t j}\right)$ according to the equipment selection $\left(Y E X_{j}\right)$, while the condition of adjacency is imposed with the auxiliary Boolean variables $W$ to define the feasible topologies.

\section{GDP-to-MILP/MINLP transformation}

A number of methods have been proposed for solving directly GDP models as disjunction problems (see Appendix 2). However, in order to take advantage of the existing MILP/MINLP solvers (see Grossmann ${ }^{[13]}$ for a review on MINLP algorithms), GDPs are often reformulated as MILP/MINLP. This section will address this procedure, which some software programs have partially automated $^{[18][22][41]}$. To describe this transformation process, the GDP formulation is partitioned into three sections as shown in Figure 7.

(GDP):

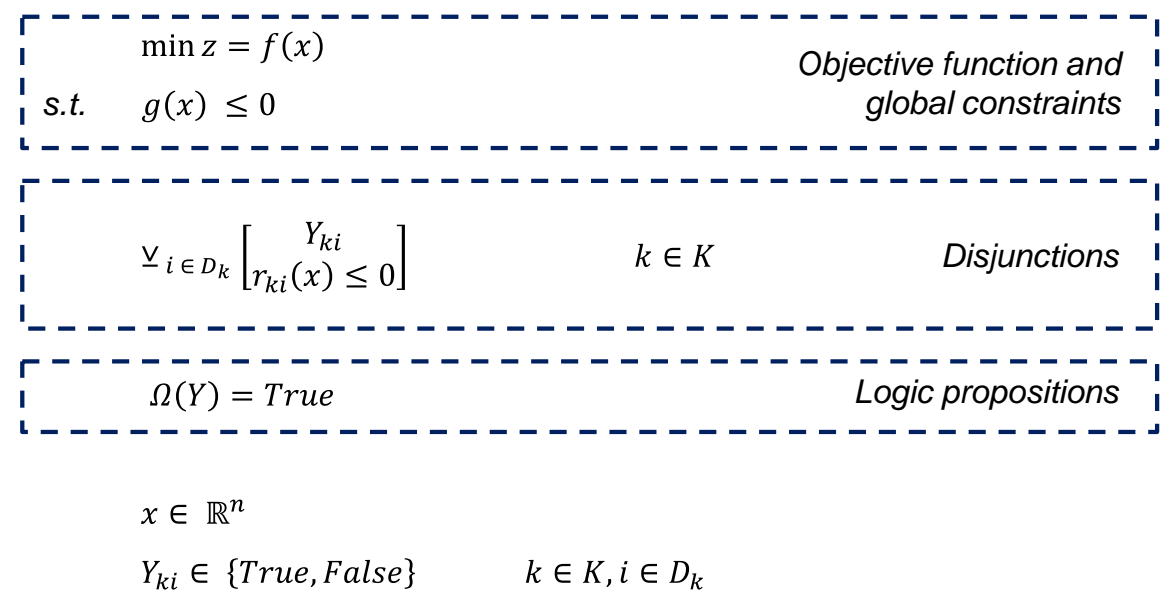

Figure 7. Partition of GDP formulation

For the first partition, the objective function and global constraints remain the same in both, the GDP and the MINLP formulations.

For the transformations of the second and third partition (disjunctions, logic propositions) it is required to convert them into algebraic form. Before we outline the detailed description of these transformations, the Boolean variables $Y_{k i}$ are converted into binary variables. To do this, binary variables $y_{k i} \in\{0,1\}$ are introduced with a one-to-one correspondence with the Boolean variables: $Y_{k i}=$ True will be transformed to $y_{k i}=1 ; Y_{k i}=$ False will be transformed to $y_{k i}=0$.

\subsection{Logic proposition into MILP constraints}

Each of the logic operators (for notation see section 2.1) can be transformed into a a set of linear $0-1$ MILP constraints as shown in Table $2^{[4][7][31][44]}$ : 
Table 2: MILP representation of Boolean connectives

\begin{tabular}{lcc}
\hline \multicolumn{1}{c}{ Logic operator } & Boolean Expression & MILP representation \\
\hline$\vee($ OR $)$ & $Y_{1} \vee Y_{2} \vee \ldots \vee Y_{n}$ & $y_{1}+y_{2}+\cdots+y_{n} \geq 1$ \\
$\underline{\vee}($ XOR $)$ & $Y_{1} \underline{\vee} Y_{2} \underline{\vee} \ldots \underline{v} Y_{n}$ & $y_{1}+y_{2}+\cdots+y_{n}=1$ \\
& & $y_{1}=1$ \\
$\wedge$ (AND) & $Y_{1} \wedge Y_{2} \wedge \ldots \wedge Y_{n}$ & $y_{2}=1$ \\
& & $\ldots$ \\
$\neg$ (NOT) & $\neg Y_{1}$ & $y_{n}=1$ \\
$\Rightarrow$ (IF) & $Y_{1} \Rightarrow Y_{2}$ & $1-y_{1}$ \\
$\Leftrightarrow$ (IF AND ONLY IF) & $Y_{1} \Leftrightarrow Y_{2}$ & $y_{1} \leq y_{2}$ \\
& & $y_{1}=y_{2}$ \\
\hline
\end{tabular}

In order to systematically derive the linear constraints with $0-1$ variables using propositional logic, the following Boolean algebra rules are used (see Williams ${ }^{[44]}$ ):

(1) Removing implication: $\quad Y_{1} \Rightarrow Y_{2} \quad \Leftrightarrow \quad \neg Y_{1} \vee Y_{2}$

(2) De Morgan's laws: $\quad \neg\left(Y_{1} \vee Y_{2}\right) \quad \Leftrightarrow \quad\left(\neg Y_{1}\right) \wedge\left(\neg Y_{2}\right)$

$\begin{array}{llll} & \neg\left(Y_{1} \wedge Y_{2}\right) & \Leftrightarrow & \left(\neg Y_{1}\right) \vee\left(\neg Y_{2}\right) \\ & \left(Y_{1} \wedge Y_{2}\right) \vee Y_{3} & \Leftrightarrow & \left(Y_{1} \vee Y_{3}\right) \wedge\left(Y_{2} \vee Y_{3}\right)\end{array}$

There are many equivalent logic forms for representing a logic proposition. One form of particular interest is the conjunctive normal form. In this form, the logic statement is represented by a conjunction of clauses $Q_{1} \wedge Q_{2} \wedge \ldots \wedge Q_{s}$ (i.e. connected by AND operators $\wedge$ ), where the clauses correspond to logic propositions that only involve the OR operator. For the conjunctive normal form to be true, each clause $Q_{i}$ must be true. With the Boolean rules described above, it is possible to systematically transform any logic proposition into its conjunctive normal form by applying recursively the following rules ${ }^{[4][8][31]}$ :

1. Replace the implication by its equivalent disjunction.

2. Move the negation inward by applying DeMorgan's rules.

3. Recursively distribute the "OR" over the "AND".

Once the logic proposition is converted into conjunctive normal form, each of the clauses $Q_{i}$ has to be satisfied, and therefore each $Q_{i}$ will translate into an inequality constraint according to the linear representation of Boolean expressions. This procedure is illustrated with the following example:

(P7): Consider the proposition ${ }^{[4]}$ :

$$
\left(Y_{1} \wedge Y_{2}\right) \vee Y_{3} \Rightarrow\left(Y_{4} \vee Y_{5}\right)
$$

1. Replace the implication by its equivalent disjunction

$$
\neg\left[\left(Y_{1} \wedge Y_{2}\right) \vee Y_{3}\right] \vee\left(Y_{4} \vee Y_{5}\right)
$$

2. Move the negation inward by applying DeMorgan's rules 


$$
\begin{aligned}
& {\left[\neg\left(Y_{1} \wedge Y_{2}\right) \wedge \neg Y_{3}\right] \vee Y_{4} \vee Y_{5}} \\
& {\left[\left(\neg Y_{1} \vee \neg Y_{2}\right) \wedge \neg Y_{3}\right] \vee Y_{4} \vee Y_{5}}
\end{aligned}
$$

3. Recursively distribute the "OR" over the "AND"

$$
\left(\neg Y_{1} \vee \neg Y_{2} \vee Y_{4} \vee Y_{5}\right) \wedge\left(\neg Y_{3} \vee Y_{4} \vee Y_{5}\right)
$$

Since (P7.5) is already in conjunctive normal form, and since it is required to satisfy each of the two clauses, the original logic proposition is equivalent to the two linear constraints in terms of the 0 - 1 variables $y_{1}, y_{2}, y_{3}, y_{4}, y_{5}$ :

$$
\begin{aligned}
& y_{1}+y_{2}-y_{4}-y_{5} \leq 1 \\
& y_{3}-y_{4}-y_{5} \leq 0
\end{aligned}
$$

Note that if we apply this procedure to the example (P2d) $y_{a} \vee y_{m} \Rightarrow\left(\neg y_{c}\right)$, we obtain the two constraints shown in (P2b) $y_{a}+y_{c} \leq 1$ and $y_{m}+y_{c} \leq 1$

\subsection{Disjunctions into MILP/MINLP constraints}

Disjunctions in GDP problems are often reformulated using either Big-M (BM) ${ }^{[30]}$ or Hull Reformulation (HR) ${ }^{[25]}$ formulations:

$\operatorname{Big}-M(B M):$

(GDP Disjunctions):

$\underline{\mathrm{v}}_{i \in D_{k}}\left[\begin{array}{c}Y_{k i} \\ r_{k i}(x) \leq 0\end{array}\right] k \in K$

\section{(BM Constraints):}

$$
\begin{array}{ll}
r_{k i}(x) \leq M^{k i}\left(1-y_{k i}\right) & k \in K, i \in D_{k} \\
\sum_{i \in D_{k}} y_{k i}=1 & k \in K
\end{array}
$$

Note that the constraint $\sum_{i \in D_{k}} y_{k i}=1$ will guarantee that for each disjunction $k \in K$ only one term $i \in D_{k}$ is active (i.e. only one binary variable $y_{k i}$ in each $k \in K$ will take a value of one). In this formulation when $y_{k i}=1$ (Boolean variable is active), the disjunction constraints $r_{k i}(x) \leq$ 0 are enforced, where $r_{k i}(x)$ can be linear or nonlinear. When $y_{k i}=0$ and the parameter $\mathrm{M}$ is significantly large, the associated constraints become redundant.

Hull-Reformulation (HR): For the Hull-Reformulation it is convenient to distinguish between the linear and nonlinear case.

Linear

(GDP Disjunctions):

$\underline{\mathrm{v}}_{i \in D_{k}}\left[\begin{array}{c}Y_{k i} \\ A^{k i} x \leq a^{k i}\end{array}\right] \quad k \in K$

\section{(HR MILP Constraints):}

$$
\begin{aligned}
& x=\sum_{i \in D_{k}} v^{k i} \\
& A^{k i} v^{k i} \leq a^{k i} y_{k i} \\
& x^{l o} y_{k i} \leq v^{k i} \leq x^{u p} y_{k i} \\
& \sum_{i \in D_{k}} y_{k i}=1
\end{aligned}
$$

$k \in K$ $k \in K, i \in D_{k}$ $k \in K, i \in D_{k}$ $k \in K$ 


$$
\begin{array}{lll} 
& x=\sum_{i \in D_{k}} v^{k i} & k \in K \\
\underline{\mathrm{v}}_{i \in D_{k}}\left[\begin{array}{c}
Y_{k i} \\
r_{k i}(x) \leq 0
\end{array}\right] k \in K & y_{k i} r_{k i}\left(v^{k i} / y_{k i}\right) \leq 0 & k \in K, i \in D_{k} \\
& x^{l o} y_{k i} \leq v^{k i} \leq x^{u p} y_{k i} & k \in K, i \in D_{k} \\
& \sum_{i \in D_{k}} y_{k i}=1 & k \in K
\end{array}
$$

In the above, the constraint $\sum_{i \in D_{k}} y_{k i}=1$ will guarantee that for each disjunction $k \in K$ only one term $i \in D_{k}$ is active (same as in (BM)). The variables $x$ are disaggregated into the variables $v^{k i}$ for each disjunctive term. An upper and lower bound constraint of the disaggregated variables associated with the binary variables is added $x^{l o} y_{k i} \leq v^{k i} \leq x^{u p} y_{k i}$, so when $y_{k i}=1$ the variable can take values between the upper and lower bound, and when $y_{k i}=0$ then $v^{k i}=0$. Also, when $y_{k i}=1$ the constraints $r_{k i}\left(v^{k i}\right) \leq 0$ are enforced; and when $y_{k i}=0$, they are trivially satisfied $(0 \leq 0)$. To avoid singularities in the nonlinear case the following approximation can be used ${ }^{[37]}$ :

$y_{k i} r_{k i}\left(v^{k i} / y_{k i}\right) \approx\left((1-\varepsilon) y_{k i}+\varepsilon\right) r_{k i}\left(\frac{v^{k i}}{(1-\varepsilon) y_{k i}+\varepsilon}\right)-\varepsilon r_{k i}(0)\left(1-y_{k i}\right)$

(APP)

where $\varepsilon$ is a small finite number (e.g. $10^{-5}$ ). This approximation is convex if $r_{k i}(x)$ is convex, and it yields an exact value of the function at $y_{k i}=0, y_{k i}=1$. It should also be noted that for linear disjunctions it is sometimes possible to eliminate the disaggregated variables by algebraic manipulation, particularly when a variable is set to zero in one of the disjunctive terms ${ }^{[32]}$.

Disjunction reformulation in model (GDP1): For the particular form (GDP1) of (GDP), the (HR) and $(\mathrm{BM})$ can be modified to provide better reformulations. The inequality in the disjunction $\hat{r}_{k i}(x) \leq 0$ is reformulated with either (BM) or (HR) as explained earlier; but the cost equality $c_{k}=\gamma_{k i}$, where $\gamma_{k i}$ is a fixed cost, is directly transformed in the objective, so the objective function $\hat{f}(x)+\sum_{k \in K} c_{k}$ becomes $\hat{f}(x)+\sum_{i \in D_{k}} \sum_{k \in K} \gamma_{i k} y_{i k}$. It is not difficult to show that this is simply the (HR) of $c_{k}=\gamma_{k i}$ after algebraic substitution. The advantage of this reformulation is that it eliminates the need of introducing disaggregated variables for $c_{k}$, while producing the (HR) of this equality. Sawaya and Grossmann ${ }^{[38]}$ provide further insight in the difference between both formulations for the linear case.

\subsection{Comparing MINLP reformulations}

In summary, by denoting $H x \geq h$ the MILP transformation of the logic propositions (obtained through the process described in 3.2), the reformulation of the linear and nonlinear GDP is as follows ${ }^{[25][30]}$ : 


\section{Linear GDP}

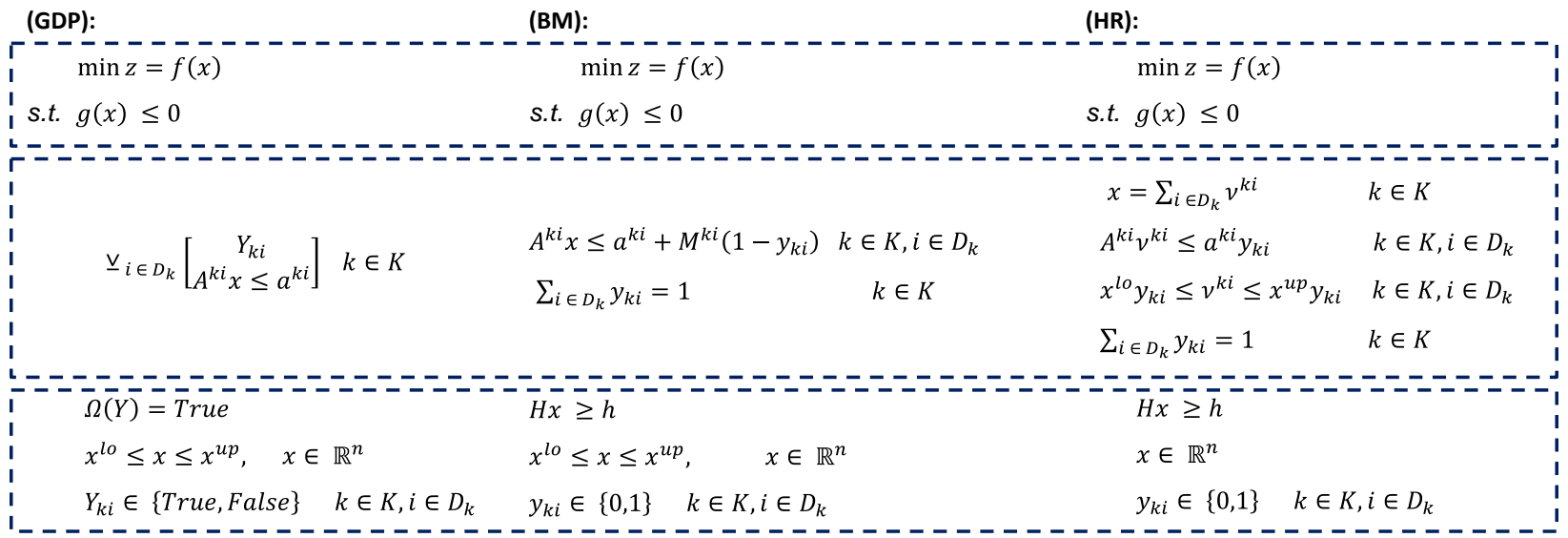

\section{$\underline{\text { Nonlinear GDP }}$}

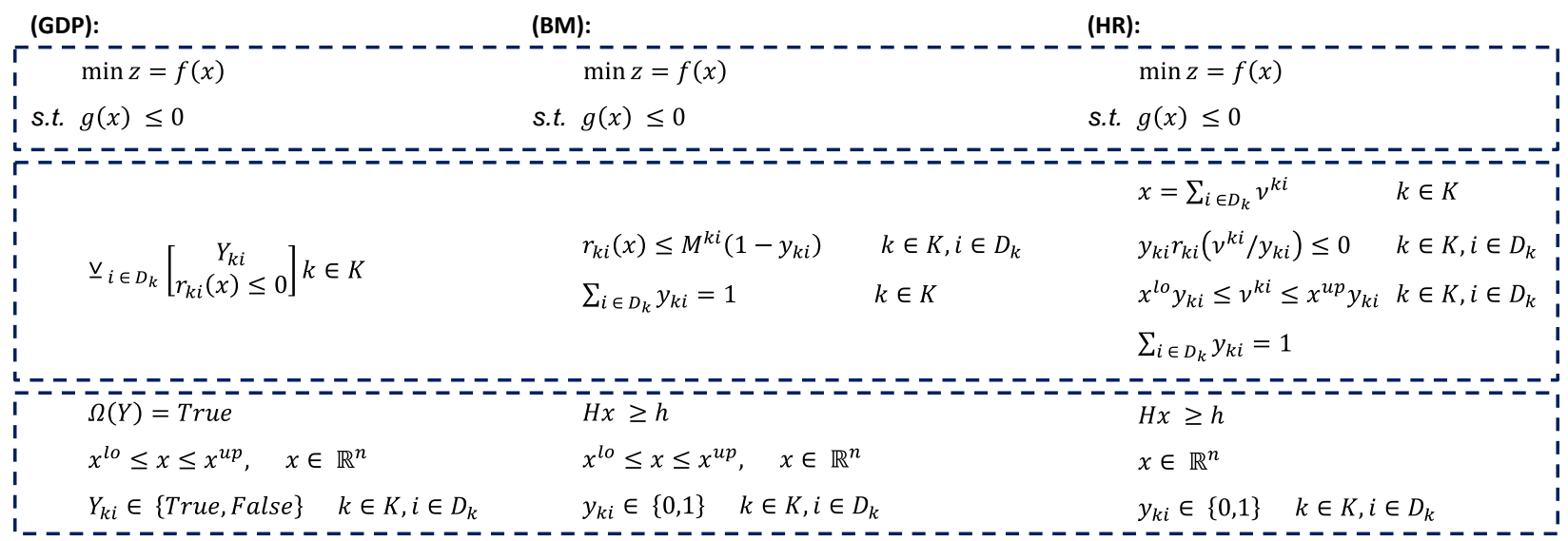

Note that because of the disaggregated variables $v^{k i}$, (HR) has more variables than BM. Additionally, in (HR) two constraints are included for each disaggregated variables (to set upper and lower limits multiplied by the binary variables), as well as the constraint that relates a variable to the disaggregated variables. Therefore, (HR) has more constraints than (BM). On the other hand, as proved in Grossmann and Lee ${ }^{[14]}$ and discussed by Vecchietti, Lee, Grossmann ${ }^{[42]}$, the continuous relaxation of the (HR) formulation is at least as tight and generally tighter than the $\mathrm{BM}$ when the discrete domain is relaxed (i.e. $0 \leq y_{k i} \leq 1 ; k \in K, i \in D_{k}$ ). This is of great importance considering that the efficiency of the MILP/MINLP solvers heavily relies on the quality of these relaxations. Figure 8 illustrate the (BM) and (HR) relaxations projected in the space of the continuous variables of a linear GDP that involves two disjunctions, each with two terms. Note that the feasible region is disjoint as it consists of the two rectangles with bold dashes. While this feasible region is the same for both formulations, the continuous relaxations are different, with the case of the (HR) clearly being tighter as it corresponds to the intersection of the convex hulls of each of the two disjunctions. 

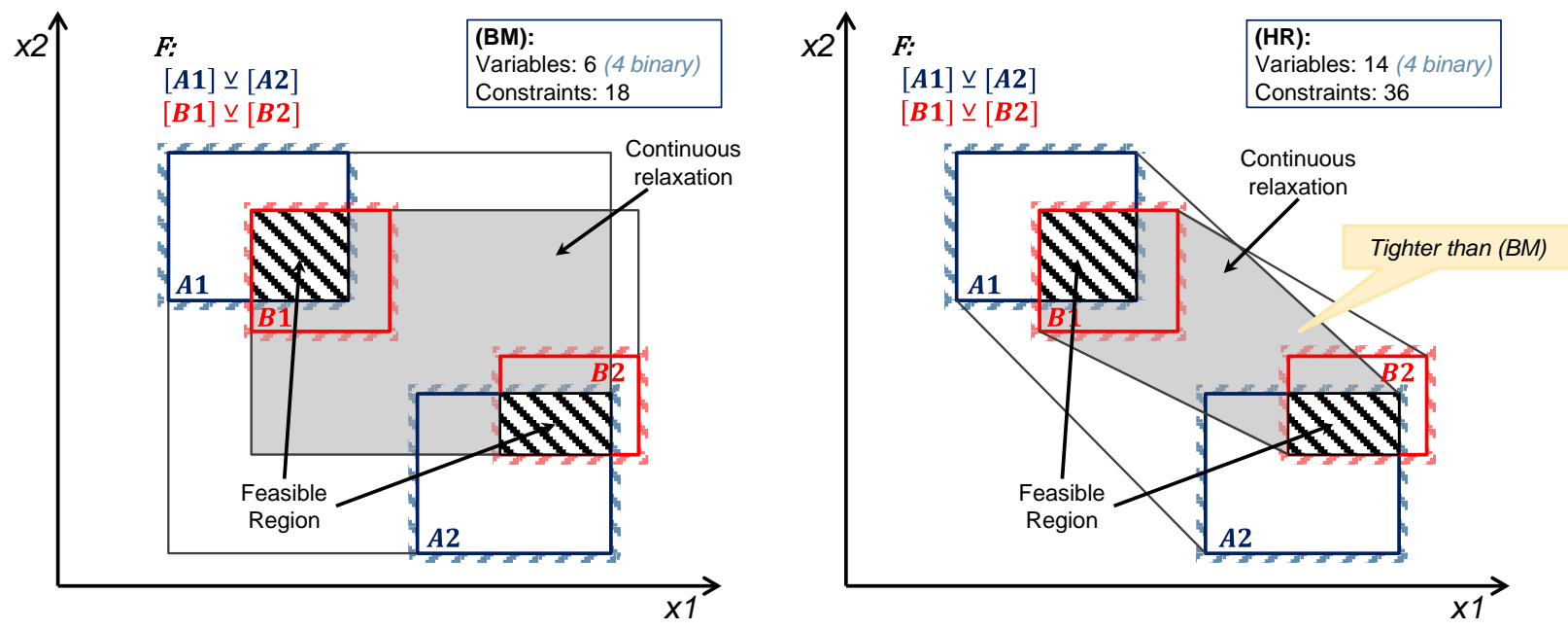

Figure 8. Illustration of Big-M and Hull Reformulation relaxation

\subsection{GDP-to-MINLP illustrations}

We illustrate the process of transforming a GDP into an MILP/MINLP with two simple examples. The first one is a linear GDP for a process synthesis problem, and the second one is the optimization of a quadratic function over three circles.

\subsubsection{Linear GDP-to-MILP: Process Synthesis Network}

Consider the process network illustrated in Figure 9, in which only one reactor and at most one separation unit can be selected. The objective is to maximize the profit of selling $F_{11}$ at a price $P_{11}$. The cost of purchasing $F_{1}$ is $P_{1}$. Each equipment has an associated cost, but unlike (GDP1), it is not constant. It can be described by a linear function representing the investment $\operatorname{cost} \gamma_{k}$ and the operating cost as a function of the flow coming out of the equipment $C_{k}=\gamma_{k}+\alpha_{k} F_{k}^{\text {out }}$.

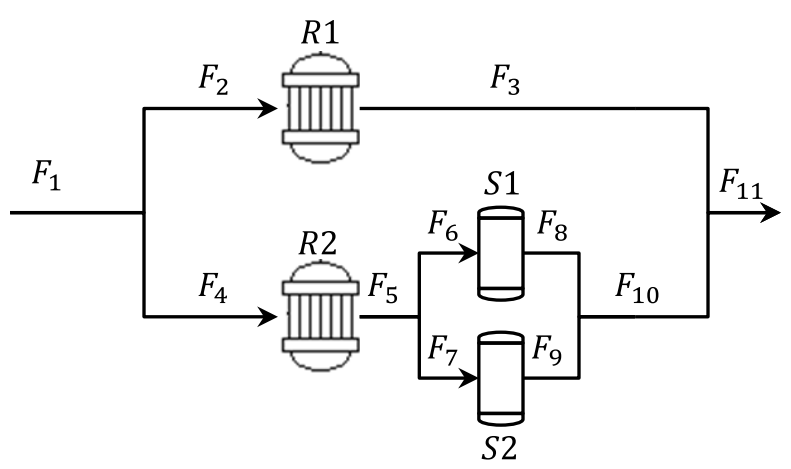

Figure 9. Illustration of a process network problem

The problem can be formulated as follows:

(P8.1):

$\max$ Profit $=P_{11} F_{11}-P_{1} F_{1}-C_{R}-C_{S}$

s.t.

$$
\begin{aligned}
& F_{1}=F_{2}+F_{4} \\
& F_{5}=F_{6}+F_{7}
\end{aligned}
$$




$$
\begin{aligned}
& \begin{array}{l}
F_{10}=F_{8}+F_{9} \\
F_{11}=F_{3}+F_{10} \\
{\left[\begin{array}{c}
Y_{R 1} \\
F_{3}=\beta_{R 1} F_{2} \\
C_{S}, F_{4,5,6,7,8,9,10}=0 \\
C_{R}=\gamma_{R 1}+\alpha_{R 1} F_{3}
\end{array}\right] \underline{v}\left[\begin{array}{c}
Y_{R 2} \\
F_{5}=\beta_{R 2} F_{4} \\
F_{2,3}=0 \\
C_{R}=\gamma_{R 2}+\alpha_{R 2} F_{5} \\
Y_{S 1} \\
F_{8}=\beta_{S 1} F_{6} \\
F_{7,9}=0 \\
C_{S}=\gamma_{S 1}+\alpha_{S 1} F_{8}
\end{array}\right] \underline{\mathrm{v}}\left[\begin{array}{c}
F_{9}=\beta_{S 2} F_{7} \\
F_{6,8}=0 \\
C_{S}=\gamma_{S 2}+\alpha_{S 2} F_{9}
\end{array}\right]} \\
\left.0 \leq F_{i} \leq F_{i}^{U P} ; 0 \leq C_{R} \leq C_{R}^{U P} ; 0 \leq C_{S} \leq C_{S}^{U P} ; Y_{R 1}, Y_{R 2}, Y_{S 1}, Y_{S 2} \in \text { True, False }\right\}
\end{array}
\end{aligned}
$$

The first step is to reformulate the problem in (GDP) form (see Appendix 1 for embedded disjunctions). Additionally, in order to obtain a smaller reformulation, in (P8.GDP) we relax the equality constraints into inequalities. It is easy to show that the problem with inequality relaxations is equivalent to the original problem.

\section{(P8.GDP):}

$$
\max \text { Profit }=P_{11} F_{11}-P_{1} F_{1}-C_{R}-C_{S}
$$

s.t.

$$
\begin{aligned}
& \begin{array}{l}
F_{1}=F_{2}+F_{4} \\
F_{5}=F_{6}+F_{7} \\
F_{10}=F_{8}+F_{9} \\
F_{11}=F_{3}+F_{10} \\
{\left[\begin{array}{c}
Y_{R 1} \\
F_{3} \leq \beta_{R 1} F_{2} \\
F_{4,5} \leq 0 \\
C_{R} \geq \gamma_{R 1}+\alpha_{R 1} F_{3}
\end{array}\right] \underline{\mathrm{v}}\left[\begin{array}{c}
Y_{R 2} \\
F_{5} \leq \beta_{R 2} F_{4} \\
F_{2,3} \leq 0 \\
C_{R} \geq \gamma_{R 2}+\alpha_{R 2} F_{5}
\end{array}\right]} \\
{\left[\begin{array}{c}
Y_{S 1} \\
F_{8} \leq \beta_{S 1} F_{6} \\
F_{7,9} \leq 0 \\
C_{S} \geq \gamma_{S 1}+\alpha_{S 1} F_{8}
\end{array}\right] \underline{\mathrm{v}}\left[\begin{array}{c}
Y_{S 2} \\
F_{9} \leq \beta_{S 2} F_{7} \\
F_{6,8} \leq 0 \\
C_{S} \geq \gamma_{S 2}+\alpha_{S 2} F_{9}
\end{array}\right] \underline{\mathrm{v}}\left[\begin{array}{c}
Y_{\text {not }} \\
C_{S}, F_{6,7,8,9,10} \leq 0
\end{array}\right]} \\
Y_{R 1} \Leftrightarrow Y_{n o t} \\
Y_{R 2} \Leftrightarrow Y_{S 1} \mathrm{v} Y_{S 2} \\
0 \leq F_{i} \leq F_{i}^{U P} ; 0 \leq C_{R} \leq C_{R}^{U P} ; 0 \leq C_{S} \leq C_{S}^{U P} ; Y_{R 1}, Y_{R 2}, Y_{n o t}, Y_{R 2}, Y_{S 2} \in\{\text { True, False }\}
\end{array}
\end{aligned}
$$

With the procedure described for the logic propositions and the (BM) or (HR) transformation to the disjunctions in sections 3.1 and 3.2, the two MILP reformulations are as follows: 
(P8.BM):

$\max$ Profit $=P_{11} F_{11}-P_{1} F_{1}-C_{R}-C_{S}$

s.t.

$$
\begin{aligned}
& F_{1}=F_{2}+F_{4} \\
& F_{5}=F_{6}+F_{7} \\
& F_{10}=F_{8}+F_{9} \\
& F_{11}=F_{3}+F_{10}
\end{aligned}
$$$$
F_{3} \leq \beta_{R 1} F_{2}+M^{R 1}\left(1-y_{R 1}\right)
$$$$
F_{4,5} \leq M^{R 1}\left(1-y_{R 1}\right)
$$$$
C_{R} \geq \gamma_{R 1}+\alpha_{R 1} F_{3}-M^{R 1}\left(1-y_{R 1}\right)
$$$$
F_{5} \leq \beta_{R 2} F_{4}+M^{R 2}\left(1-y_{R 2}\right)
$$$$
F_{2,3} \leq M^{R 2}\left(1-y_{R 2}\right)
$$$$
C_{R} \geq \gamma_{R 2}+\alpha_{R 2} F_{5}-M^{R 2}\left(1-y_{R 2}\right)
$$$$
F_{8} \leq \beta_{S 1} F_{6}+M^{S 1}\left(1-y_{S 1}\right)
$$$$
F_{7,9} \leq M^{S 1}\left(1-y_{S 1}\right)
$$$$
C_{S} \geq \gamma_{S 1}+\alpha_{S 1} F_{8}-M^{S 1}\left(1-y_{S 1}\right)
$$$$
F_{9} \leq \beta_{S 2} F_{7}+M^{S 2}\left(1-y_{S 2}\right)
$$$$
F_{6,8} \leq M^{S 2}\left(1-y_{S 2}\right)
$$$$
C_{S} \geq \gamma_{S 2}+\alpha_{S 2} F_{9}-M^{S 2}\left(1-y_{S 2}\right)
$$$$
C_{S}, F_{6,7,8,9,10} \leq M^{n o t} R_{R 2}\left(1-y_{n o t}\right)
$$$$
y_{R 1}+y_{R 2}=1
$$$$
y_{S 1}+y_{S 2}+y_{n o t}=1
$$$$
y_{R 1}=y_{n o t}
$$$$
y_{R 2}=y_{S 1}+y_{S 2}
$$$$
0 \leq F_{i} \leq F_{i}^{U P} \quad i=1, \ldots, 11
$$$$
0 \leq C_{R} \leq C_{R}^{U P}
$$$$
0 \leq C_{S} \leq C_{S}^{U P}
$$

$y_{k} \in\{0,1\} ; \quad k \in\left\{R 1, R 2, S 1, S 2, \operatorname{not}_{R 2}\right\}$

\section{(P8.HR):}

$\max$ Profit $=P_{11} F_{11}-P_{1} F_{1}-C_{R}-C_{S}$

s.t.

$$
\begin{aligned}
& F_{1}=F_{2}+F_{4} \\
& F_{5}=F_{6}+F_{7} \\
& F_{10}=F_{8}+F_{9} \\
& F_{11}=F_{3}+F_{10}
\end{aligned}
$$$$
\begin{array}{ll}
F_{i}=F_{i}^{R 1}+F_{i}^{R 2} & i=1, \ldots, 11 \\
F_{i}=F_{i}^{S 1}+F_{i}^{S 2}+F_{i}^{n o t} t_{R 2} & i=1, \ldots, 11 \\
C_{R}=C_{R}^{R 1}+C_{R}^{R 2} & \\
C_{S}=C_{S}^{S 1}+C_{S}^{S 2}+C_{S}^{n o t} t_{R 2} &
\end{array}
$$$$
F_{3}^{R 1} \leq \beta_{R 1} F_{2}^{R 1}
$$$$
F_{4,5}^{R 1} \leq 0
$$$$
C_{R}^{R 1} \geq \gamma_{R 1} y_{R 1}+\alpha_{R 1} F_{3}^{R 1}
$$$$
F_{5}^{R 2} \leq \beta_{R 2} F_{4}^{R 2}
$$$$
F_{2,3}^{R 2} \leq 0
$$$$
C_{R}^{R 2} \geq \gamma_{R 2} y_{R 2}+\alpha_{R 2} F_{5}^{R 2}
$$$$
F_{8}^{S 1} \leq \beta_{S 1} F_{6}^{S 1}
$$$$
F_{7,9}^{S 1} \leq 0
$$$$
C_{S}^{S 1} \geq \gamma_{S 1} y_{S 1}+\alpha_{S 1} F_{8}^{S 1}
$$$$
F_{9}^{S 2} \leq \beta_{S 2} F_{7}^{S 2}
$$$$
F_{6,8}^{S 2} \leq 0
$$$$
C_{S}^{S 2} \geq \gamma_{S 2} y_{S 2}+\alpha_{S 2} F_{9}^{S 2}
$$$$
C_{S}^{n o t_{R 2}}, F_{6,7,8,9,10}^{n o t} \leq 0
$$

$y_{R 1}+y_{R 2}=1$

$y_{S 1}+y_{S 2}+y_{\text {not }_{R 2}}=1$

$y_{R 1}=y_{n o t_{R 2}}$

$y_{R 2}=y_{S 1}+y_{S 2}$

$\left.0 \leq F_{i}^{k} \leq F_{i}^{U P} y_{k}\right] \quad i=1, \ldots, 11$;

$0 \leq C_{R}^{k} \leq C_{R}^{U P} y_{k} \quad k \in\left\{R 1, R 2, S 1, S 2\right.$, not $\left._{R 2}\right\}$

$0 \leq C_{S}^{k} \leq C_{S}^{U P} y_{k}$

$y_{k} \in\{0,1\} ; \quad k \in\left\{R 1, R 2, S 1, S 2, \operatorname{not}_{R 2}\right\}$ 
As can be seen these two alternative formulations have been derived systematically from the GDP (P8.1). Thus, we have avoided a direct ad-hoc formulation of this problem in algebraic form as an MILP which may or may not correspond to a "good" formulation. Having the two models (P8.BM) and (P8.HR) we have a better understanding and insight of what we can expect from each formulation, although clearly the numerical performance will depend on the actual parameter of the given instance.

\subsubsection{Nonlinear GDP-to-MINLP: Quadratic function over three circles ${ }^{[25]}$}

Consider the analytical problem given in Lee and Grossmann ${ }^{[25]}$ which consists in finding the minimum of a quadratic objective function, subject to a disjoint feasible region defined by circles as shown in Figure 10. The problem formulation is given by (P9.GDP).

(P9.GDP):

$\min Z=\left(x_{1}-5\right)^{2}+\left(x_{2}-5\right)^{2}$

st.

$$
\begin{aligned}
& {\left[\begin{array}{c}
Y_{1} \\
x_{1}^{2}+x_{2}^{2} \leq 1
\end{array}\right] \underline{\mathrm{v}}\left[\begin{array}{c}
Y_{2} \\
\left(x_{1}-4\right)^{2}+\left(x_{2}-1\right)^{2} \leq 1
\end{array}\right] \underline{\mathrm{v}}\left[\begin{array}{c}
Y_{3} \\
\left(x_{1}-2\right)^{2}+\left(x_{2}-4\right)^{2} \leq 1
\end{array}\right]} \\
& -5 \leq x_{1}, x_{2} \leq 5 ; Y_{1}, Y_{2}, Y_{3} \in\{\text { True, False }\}
\end{aligned}
$$

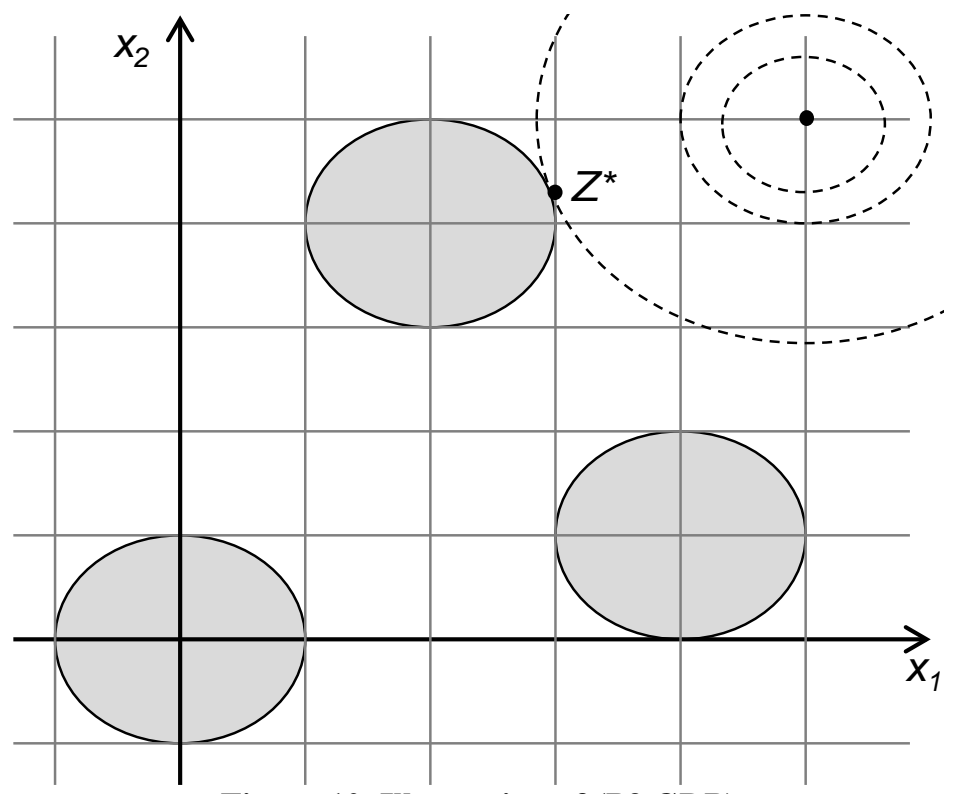

Figure 10. Illustration of (P9.GDP)

(P9.BM) and (P9.HR) show the corresponding MINLP transformation. For the case of the (HR) transformation we use the approximation (APP) ${ }^{[37]}$.

(P9.BM):

$$
\min Z=\left(x_{1}-5\right)^{2}+\left(x_{2}-5\right)^{2}
$$

st. 


$$
\begin{aligned}
& x_{1}^{2}+x_{2}^{2} \leq 1+M\left(1-y_{1}\right) \\
& \left(x_{1}-4\right)^{2}+\left(x_{2}-1\right)^{2} \leq 1+M\left(1-y_{2}\right) \\
& \left(x_{1}-2\right)^{2}+\left(x_{2}-4\right)^{2} \leq 1+M\left(1-y_{3}\right) \\
& y_{1}+y_{2}+y_{3}=1 \\
& -5 \leq x_{1}, x_{2} \leq 5 \\
& y_{1,2} \in\{0,1\}
\end{aligned}
$$

\section{(P9.HR):}

st.

$$
\min Z=\left(x_{1}-5\right)^{2}+\left(x_{2}-5\right)^{2}
$$

$$
\begin{aligned}
& x_{1}=x_{1 \_1}+x_{1 \_2}+x_{1 \_3} \\
& x_{2}=x_{2 \_1}+x_{2 \_2}+x_{2 \_3} \\
& \left((1-\varepsilon) y_{1}+\varepsilon\right)\left[\left(\frac{x_{1 \_1}}{(1-\varepsilon) y_{1}+\varepsilon}\right)^{2}+\left(\frac{x_{2 \_1}}{(1-\varepsilon) y_{1}+\varepsilon}\right)^{2}-1\right]+\varepsilon\left(1-y_{1}\right) \leq 0 \\
& \left((1-\varepsilon) y_{2}+\varepsilon\right)\left[\left(\frac{x_{1 \_2}}{(1-\varepsilon) y_{2}+\varepsilon}-4\right)^{2}+\left(\frac{x_{2 \_}}{(1-\varepsilon) y_{2}+\varepsilon}-1\right)^{2}-1\right]-16 \varepsilon\left(1-y_{2}\right) \leq 0 \\
& \left((1-\varepsilon) y_{3}+\varepsilon\right)\left[\left(\frac{x_{1 \_3}}{(1-\varepsilon) y_{3}+\varepsilon}-2\right)^{2}+\left(\frac{x_{2 \_}}{(1-\varepsilon) y_{3}+\varepsilon}-4\right)^{2}-1\right]-19 \varepsilon\left(1-y_{3}\right) \leq 0 \\
& y_{1}+y_{2}+y_{3}=1 \\
& -5 y_{i} \leq x_{j_{-} i} \leq 5 y_{i} \quad i=1,2,3 ; j=1,2 \\
& y_{1,2} \in\{0,1\}
\end{aligned}
$$

The optimal solution to (P9.GDP) is 4.68. If the continuous relaxations of (P9.BM) and (P9.HR) are considered by solving the corresponding NLPs, the respective lower bounds 0.45 and 4.20 are obtained, showing that the (HR) provides a stronger lower bound since it has a tighter relaxation.

\subsection{Improving convex GDP reformulations through Basic Steps}

While the HR formulation provides tighter formulation than (BM), it is possible to develop formulations that are even tighter than HR using some basic concepts of disjunctive programming $^{[1][2]}$.

GDP can be regarded as an extension of the well-known Disjunctive Programming model developed by Balas ${ }^{[1][2]}$, and which consists of a linear program with disjunctions, but no Boolean variables. Sawaya and Grossmann ${ }^{[37][38]}$ proved that any Linear (GDP) can be equivalently formulated as a Disjunctive Program, and therefore the rich theory behind Disjunctive Programming can be used in order to solve Linear GDPs more efficiently. Ruiz and 
Grossmann $^{[33][35]}$ extended this theory to nonlinear convex GDP. A convex GDP is a particular case of (GDP) in which the functions $f(x), g(x)$ and $r_{k i}(x)$ are convex.

A particular logic operation in disjunctive programming that helps to generate a tighter formulation than the (HR) of the problem is the so-called Basic Step ${ }^{[2]}$.

\subsubsection{Basic Step in Disjunctive Programming ${ }^{[1][2][35]}$}

To describe this operation it is important to consider the following definitions ${ }^{[2][35] \text { : }}$

Convex inequality: (Halfspace in linear case)

$H=\left\{x \in \mathbb{R}^{n} \mid \phi(x) \leq 0\right\}$, where $\phi(x): \mathbb{R}^{n} \rightarrow \mathbb{R}^{1}$ is a convex function

Convex set: (Polyhedron in linear case)

$P=\bigcap_{i \in M} H_{i}$

Elementary disjunctive set:

$D=\bigcup_{i \in M} H_{i}$

A disjunctive set can be expressed in many different logically equivalent forms. Three of them are of particular interest:

Conjunctive Normal Form ( $\underline{\mathrm{CNF}}): F=\bigcap_{i \in T} D_{i}=\bigcap_{i \in T} \bigcup_{i \in M} H_{i}$

Disjunctive Normal Form (DNF): $F=\bigcup_{i \in Q} P_{i}=\bigcup_{i \in Q} \bigcap_{i \in M} H_{i}$

Regular Form (ㅌ) (intersection of DNFs): $F=\bigcap_{j \in T} S_{j}$, where $S_{j}=\bigcup_{i \in Q} P_{i}$

Note that the CNF and DNF are two extremes of equivalent forms, while RF is in between them. Also note that while any 0-1 MILP/MINLP is in CNF, the GDP is in RF.

As stated and proved in Theorem 2.1 of Balas ${ }^{[2]}$, any disjunctive set in Regular Form can be converted into DNF by a recursive application of an operation called Basic Step. In particular, given a disjunctive set in RF, it can be brought to DNF by $|\mathrm{T}|-1$ recursive applications of the following basic step, that involves intersecting pairs of disjunctions. That is, for some $k, l \in T, k$ $\neq l, S_{k} \cap S_{l}$ is brought to DNF by replacing it with $S_{k l}=U_{i \in Q_{k}, j \in Q_{l}}\left(P_{i} \cap P_{j}\right)$.

Additionally, as stated and proved in Theorem 4.3 of Balas ${ }^{[2]}$, the hull relaxation of a disjunctive set $\operatorname{hrel}(F):=\bigcap_{j \in T} \operatorname{cl} \operatorname{conv}\left(S_{j}\right)$, where $\operatorname{cl} \operatorname{conv}$ is the closed convex set, after the application of a basic step is as tight, if not tighter, than the previous hull relaxation. It follows that the hull relaxation of a disjunctive set in DNF is equal to its convex hull (or perfect formulation, since it can be solved as a continuous optimization problem).

It is important to note that when a basic step is applied, the hull relaxation of the new disjunctive set has more continuous variables and constraints. There are different rules that have been proposed for deciding when to apply or not a basic step ${ }^{[2][35][37]}$. 


\subsubsection{Illustrative example of Basic Step in GDP}

A Basic Step is illustrated with the following example: The objective is to maximize profit by selling a product $\mathrm{P}$ at a price 10 . To produce it, it is required to buy either reactor $\mathrm{R} 1$ with cost $\mathrm{C}=5 *$ inlet flow and $90 \%$ conversion of raw material $\mathrm{A}$, or reactor $\mathrm{R} 2$ with $\mathrm{C}=4.6 *$ inlet flow and $80 \%$ conversion of raw material $\mathrm{B}$. The cost of raw material $\mathrm{A}$ is 1.1 and of $\mathrm{B}$ is 1 . The maximum allowed cost for any selected equipment is 30 , and we can select only one of the raw materials. There is a limit on the availability, for $\mathrm{A}=5$, and $\mathrm{B}=7$. The GDP can be formulated as follows:

(P10.1):

$\max$ Profit $=10 F_{P}-C_{e q}-C_{\text {raw }}$

s.t.

$\left[\begin{array}{c}Y_{R 1} \\ F_{P}=0.9 F_{A} \\ C_{e q}=5.0 F_{A}\end{array}\right] \underline{\mathrm{v}}\left[\begin{array}{c}Y_{R 2} \\ F_{P}=0.8 F_{B} \\ C_{e q}=4.6 F_{B}\end{array}\right]$

$\left[\begin{array}{c}Y_{\text {Raw } A} \\ C_{\text {raw }}=1.1 F_{A} \\ F_{B}=0\end{array}\right] \underline{v}\left[\begin{array}{c}Y_{\text {RawB }} \\ C_{\text {raw }}=1 F_{B} \\ F_{A}=0\end{array}\right]$

$0 \leq F_{A} \leq 5$

$0 \leq F_{B} \leq 7$

$0 \leq C_{e q} \leq 30$

$Y_{R 1}, Y_{R 2}, Y_{\text {RawA }}, Y_{\text {RawB }} \in\{$ True, False $\}$

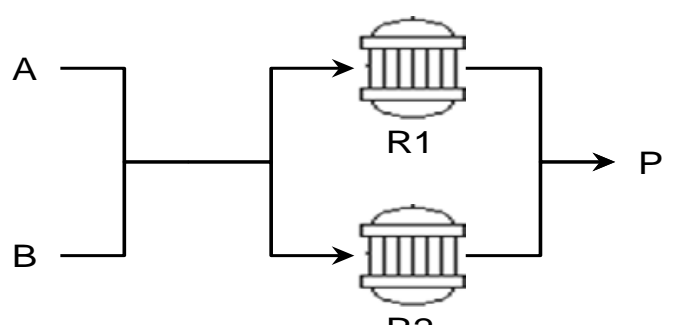

R2

Figure 11: Structure of Reactor and raw material selection in (P10.1)

Note that the problem has 2 proper disjunctions (those that contain the XOR operator), with two terms each disjunction, and two equations each term. It also contains 6 improper disjunctions (the upper and lower bounds of $F_{A}, F_{B}$ and $C_{e q}$, i.e. the global constraints). The solution to this problem is to select reactor 2 and purchase raw material $\mathrm{B}$, with profit $=1$ 15.7. For the relaxation of (BM) the profit $=\mathbf{5 0 , 0 5 0}$, and for the relaxation of (HR), the profit $=\mathbf{1 6 . 1}$, which is clearly much tighter.

Intersecting the two disjunctions, which represents performing a basic step, and distributing the XOR over the AND operator, leads to the following problem:

\section{(P10.2)}

$$
\max \text { Profit }=10 F_{P}-C_{e q}-C_{\text {raw }}
$$

s.t.

$$
\begin{aligned}
& {\left[\begin{array}{c}
Y_{R 1} A N D Y_{\text {RawA }} \\
F_{P}=0.9 F_{A} \\
C_{e q}=5.0 F_{A} \\
C_{\text {raw }}=1.1 F_{A} \\
F_{B}=0
\end{array}\right] \underline{\mathrm{v}}\left[\begin{array}{c}
Y_{R 1} A N D Y_{\text {RawB }} \\
F_{P}=0.9 F_{A} \\
C_{e q}=5.0 F_{A} \\
C_{\text {raw }}=1 F_{B} \\
F_{A}=0
\end{array}\right] \underline{\mathrm{v}}\left[\begin{array}{c}
Y_{R 2} A N D Y_{\text {RawA }} \\
F_{P}=0.8 F_{B} \\
C_{e q}=4.6 F_{B} \\
C_{\text {raw }}=1.1 F_{A} \\
F_{B}=0
\end{array}\right] \underline{\mathrm{v}}\left[\begin{array}{c}
Y_{R 2} A N D Y_{\text {RawB }} \\
F_{P}=0.8 F_{B} \\
C_{e q}=4.6 F_{B} \\
C_{\text {raw }}=1 F_{B} \\
F_{A}=0
\end{array}\right]} \\
& 0 \leq F_{A} \leq 5 \\
& 0 \leq F_{B} \leq 7 \\
& 0 \leq C_{\text {eq }} \leq 30 \\
& Y_{R 1}, Y_{R 2}, Y_{\text {RawA }}, Y_{\text {Raw }} \in\{\text { True, False }\}
\end{aligned}
$$


It is easy to see that (P10.1) and (P10.2) are logically equivalent, since (P10.2) is only enumerating the potential combinations between R1 and R2 with raw materials $\mathrm{A}$ and $\mathrm{B}$. Also note that the problem now contains a single proper disjunction, with 4 disjunctive terms, and 5 equations each term. This "distribution” operation is called proper Basic Step. The application of this operation to the improper disjunctions (global constraints), is called an improper Basic Step. The extreme case in which the problem is defined over a single disjunction is called Disjunctive Normal Form. The DNF of (P10.1) is then as follows:

$$
\begin{aligned}
& \text { (P10.3 = DNF) } \\
& \text { max Profit }=10 F_{P}-C_{e q}-C_{\text {raw }} \\
& \text { s.t. } \\
& {\left[\begin{array}{c}
Y_{R 1} A N D Y_{R a w A} \\
F_{P}=0.9 F_{A} \\
C_{e q}=5.0 F_{A} \\
C_{r a w}=1.1 F_{A} \\
F_{B}=0 \\
0 \leq F_{A} \leq 5 \\
0 \leq F_{B} \leq 7 \\
0 \leq C_{e q} \leq 7
\end{array}\right] \underline{\mathrm{v}}\left[\begin{array}{c}
Y_{R 1} A N D Y_{R a w B} \\
F_{P}=0.9 F_{A} \\
C_{e q}=5.0 F_{A} \\
C_{\text {raw }}=1 F_{B} \\
F_{A}=0 \\
0 \leq F_{A} \leq 5 \\
0 \leq F_{B} \leq 7 \\
0 \leq C_{e q} \leq 7
\end{array}\right] \underline{\mathrm{v}}\left[\begin{array}{c}
Y_{R 2} A N D Y_{R a w A} \\
F_{P}=0.8 F_{B} \\
C_{e q}=4.6 F_{B} \\
C_{r a w}=1.1 F_{A} \\
F_{B}=0 \\
0 \leq F_{A} \leq 5 \\
0 \leq F_{B} \leq 7 \\
0 \leq C_{e q} \leq 7
\end{array}\right] \underline{\mathrm{v}}\left[\begin{array}{c}
Y_{R 2} A N D Y_{\text {RawB }} \\
F_{P}=0.8 F_{B} \\
C_{e q}=4.6 F_{B} \\
C_{\text {raw }}=1 F_{B} \\
F_{A}=0 \\
0 \leq F_{A} \leq 5 \\
0 \leq F_{B} \leq 7 \\
0 \leq C_{e q} \leq 7
\end{array}\right]} \\
& Y_{R 1}, Y_{R 2}, Y_{\text {RawA }}, Y_{\text {RawB }} \in\{\text { True }, \text { False }\}
\end{aligned}
$$

It is also clear to see the logic equivalence of (P10.2) and (P10.3), only that in (P10.3) the global constraints are inside the disjunctive terms. The relaxation of the (HR) of (P10.3) yields a profit = 15.7, which is the same as the optimal MILP solution. Note that with the application of $|\mathrm{K}|-1$ Basic Steps a problem achieves DNF ${ }^{[35][15]}$ form.

There are three main consequences of this operation ${ }^{[2]}$ : i) The size of the problem increases as we apply BS (exponentially in terms when applying proper Basic Steps, and increase in the number of constraints when applying improper Basic Steps); ii) The problem generated after applying a BS is at least as tight as before, and possibly tighter; iii) The HR of the DNF is a perfect formulation. For a recent algorithmic implementation of these concepts see [39]

\subsubsection{Extension of Basic Step to Global Optimization of Nonconvex GDP}

A discussion on global optimization of nonconvex GDP problems is out of the scope of this paper. However, to provide some context we include the following brief description. In order to apply the theory of Disjunctive Programming into nonconvex GDP for global optimization, Ruiz and Grossmann ${ }^{[34]}$ proposed a two stage approach. The first stage relaxes the nonconvexities by replacing them with over and under estimating functions, or convex envelopes, such as the ones by McCormick ${ }^{[27]}$ for bilinear terms. Figure 12 shows the general framework of this approach, which has the net result of providing stronger lower bounds for the global optimum solution of the nonconvex GDP. This is important because the effectiveness of the global optimization method relies heavily on the quality of this bound. 


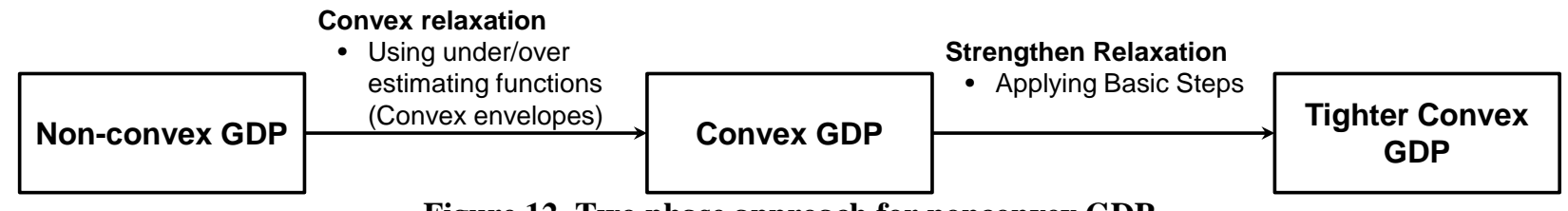

Figure 12. Two phase approach for nonconvex GDP

\section{Numerical examples}

In this section we compare the different formulations and improvement after the application of Basic Steps (according the corresponding reference) for three examples of modest size taken from the literature. The first example (P11) is linear; the next is nonlinear but convex (P12); and the final example is nonconvex (P13). For the nonconvex problem, problem size refers to the MINLP reformulation of the convex GDP relaxation.

\subsection{Linear GDP: Strip-packing Problem ${ }^{[38]}$}

A given set of rectangles is to be packed into a strip of fixed width $\mathrm{W}$ but unknown length $\mathrm{L}$. The objective is to minimize the length of the strip while fitting all rectangles without any overlap and without rotation. The GDP formulation is as follows ${ }^{[38]}$ :

(P11):

$\min l t$

st. $\quad l t \geq x_{i}+L_{i}$

$i \in N$

$$
\begin{aligned}
& {\left[\begin{array}{c}
Y_{i j}^{1} \\
x_{i}+L_{i} \leq x_{j}
\end{array}\right] \underline{\mathrm{v}}\left[\begin{array}{c}
Y_{i j}^{2} \\
x_{j}+L_{j} \leq x_{i}
\end{array}\right] \underline{\mathrm{v}}\left[\begin{array}{c}
Y_{i j}^{3} \\
y_{i}-H_{i} \geq y_{j}
\end{array}\right] \underline{\mathrm{v}}\left[\begin{array}{c}
Y_{i j}^{4} \\
y_{j}-H_{j} \geq y_{i}
\end{array}\right] \quad i, j \in N, i<j} \\
& 0 \leq x_{i} \leq U B-L_{i} \quad i \in N \\
& H_{i} \leq y_{i} \leq W \quad i \in N \\
& l t, x_{i}, y_{i} \in \mathbb{R}^{1}, Y_{i k} \in\{\text { True, False }\} \quad i, j \in N, i<j
\end{aligned}
$$

where $x_{i}$ and $y_{i}$ represent the coordinates of the upper-left corner of the rectangles $i \in N$. There is one disjunction for each pair of rectangles (e.g. if there are 4 rectangles there will be 6 disjunctions representing the combinations of rectangles $(1,2),(1,3),(1,4),(2,3),(2,4),(3,4))$. Each term in a disjunction represents the possible relative placement of the rectangles to ensure that they will not overlap. Specifically, the four terms represent that rectangle $i$ is either to the right or to the left, or above or below rectangle $j$, respectively:

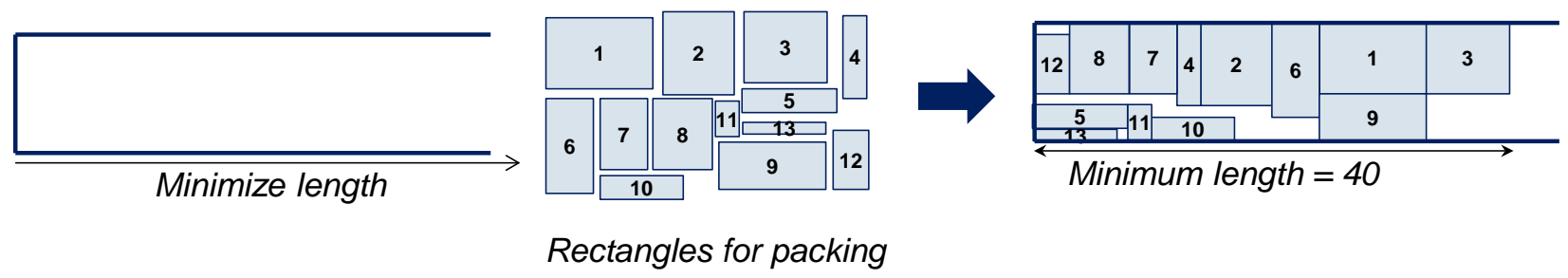

Figure 13. Strip packing illustration for a 13 rectangle problem 
The example presented in Sawaya et $\mathrm{al}^{[38]}$ consists of 8 rectangles with the dimensions shown in Table 3 and with a Width of 10 .

Table 3: Dimensions for 8-rectangle strip-packing problem.

\begin{tabular}{ccc}
\hline Rectangle & Length & Height \\
\hline 1 & 4 & 3 \\
2 & 3 & 3 \\
3 & 2 & 2 \\
4 & 2 & 2 \\
5 & 3 & 3 \\
6 & 3 & 5 \\
7 & 4 & 7 \\
8 & 4 & 7 \\
\hline
\end{tabular}

The solution, size of the problem and tightness of the relaxation as reported in [38] is shown in Table 4. The MILP solver used was CPLEX.

Table 4: Results for 8-rectangle strip-packing problem.

\begin{tabular}{lcccc}
\hline & $(\mathrm{BM})$ & $(\mathrm{HR})$ & $(\mathrm{HR})$ after Basic Steps & (HR) in DNF \\
\hline Optimal solution & 11 & 11 & 11 & 11 \\
Relaxation & $\mathbf{4}$ & $\mathbf{6}$ & $\mathbf{1 1}$ & $\mathbf{1 1}$ \\
Number of constraints & 168 & 862 & 932 & 344,081 \\
Number of variables & 123 & 535 & 563 & 147,473 \\
Number of binaries & 106 & 106 & 106 & - \\
Number of nodes & 212,464 & 2,596 & 1,464 & 0 \\
Solution time (s) & 13.7 & 2.5 & 1.6 & $>3,600$ \\
\hline \multicolumn{1}{l}{ 1. Selection of disjunctions to apply the basic steps is discussed in source }
\end{tabular}

It is clear from Table 4 that the size of the problem is considerably larger in (HR) than in (BM). It is also possible to observe that the application of some Basic Steps leads to a major improvement in the relaxation, yielding the optimal solution of 11, and increasing slightly the size of the problem. It is important to mention that the authors performed a preprocessing to select in which disjunctions to apply the basic steps, and such preprocessing would require some execution time. Finally, although the problem in explicit DNF form represents the convex-hull of the problem ${ }^{[2]}$ (i.e. its continuous relaxed solution will always provide an integral value for the discrete variables), the size of the problem makes it impossible to solve it in less than 3,600 seconds using the MILP solver CPLEX.

\subsection{Non-linear convex GDP: Process Network ${ }^{[35]}$}

In general, the underlying goal of a classical process synthesis problem consists in selecting the process that maximizes the profit when selling a product or set of products considering the cost of raw materials and the cost of the process units. The model in the form of the GDP problem involves disjunctions for the selection of units, and propositional logic for the relationship of these units. Each disjunction contains the equation for each unit (these relax as convex inequalities). This example is a particular instance with twelve unit operations as illustrated in Figure 14. A general GDP model is shown in (P12): 


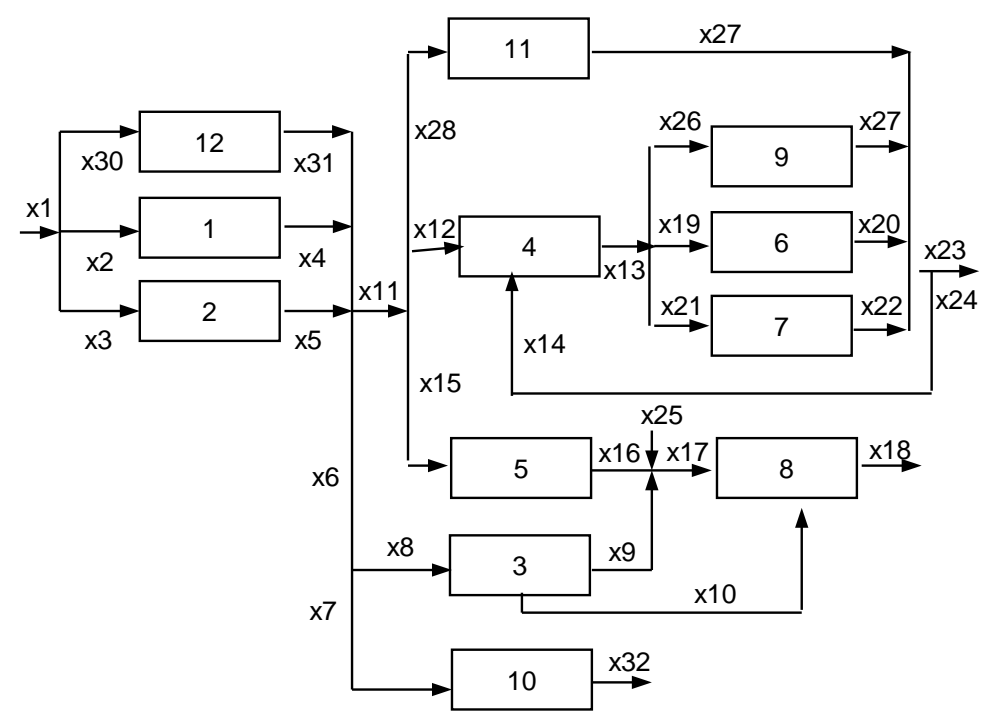

Figure 14. Process Network illustration for a 12-unit instance

(P12):

$\min \sum_{i=1}^{12} c_{i}+\sum_{j=1}^{32} p_{j} x_{j}+\gamma$

st.

$\sum_{j=1}^{32} r_{j n} x_{j} \leq 0 \quad \forall n \in \mathrm{N}$

$\left[\begin{array}{c}Y_{i} \\ \sum_{j=1}^{32} d_{i j}\left(e^{x_{j} / t_{i j}}-1\right)-\sum_{j=1}^{32} s_{i j} x_{j} \leq 0 \\ c_{i}=\gamma_{i}\end{array}\right] \underline{\mathrm{v}}\left[\begin{array}{c}\neg Y_{i} \\ x_{j}=0 ; j \in J^{i} \\ c_{i}=0\end{array}\right] \quad i=1, \ldots, 12$

$\Omega(Y)=$ True

$x_{j}, c_{i} \geq 0 \quad i=1, \ldots, 12$

$Y_{i} \in\{$ True, False $\}$

The solution, size of the problem and tightness of the relaxation as reported in [35] is shown in Table 5. The MINLP solver used was SBB.

Table 5: Results for 12-unit process network problem.

\begin{tabular}{lccc}
\hline & $(\mathrm{BM})$ & $(\mathrm{HR})$ & $(\mathrm{HR})$ after Basic Steps $^{1}$ \\
\hline Optimal solution & -69.5 & -69.5 & -69.5 \\
Relaxation & $\mathbf{- 1 , 1 0 8 . 9}$ & $\mathbf{- 7 4 . 8}$ & $\mathbf{- 6 9 . 5}$ \\
Number of constraints & 114 & 184 & 1,462 \\
Number of variables & 69 & 149 & 807 \\
Number of binaries & 12 & 12 & 12 \\
Number of nodes & 234 & 8 & 2 \\
Solution time (s) & 27.7 & 1.0 & 2.9 \\
\hline
\end{tabular}

1. Selection of disjunctions to apply the basic steps is discussed in source 
As shown in Table 5, it is clear that (HR) has much better relaxation than (BM). In this example, the application of Basic Steps slightly improves the relaxation of (HR), yielding in fact the optimal solution. Furthermore, it also reduces the number of nodes in the MINLP branch and bound method (SBB was used). However, the problem size grows considerably, although the computational time is quite reasonable

\subsection{Nonconvex GDP: Heat Exchange Network (HEN) ${ }^{[34]}$}

This problem consists of finding the heat loads of utilities, the intermediate temperatures and the area of each exchanger that minimizes the investment and operation cost. In this case the investment cost is given by a discontinuous cost function defined over three intervals in terms of the area. Note that the structure of the network is fixed and that we use the arithmetic mean temperature as the driving force. Figure 15 illustrates the HEN structure.

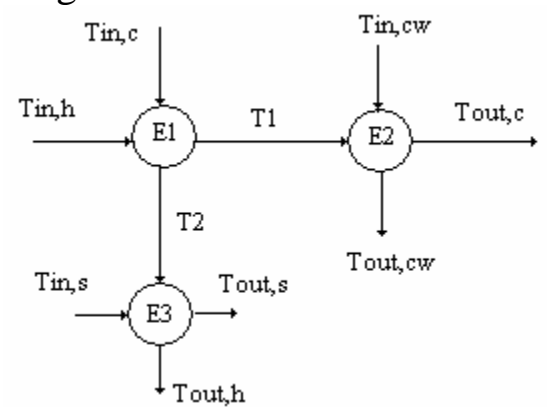

Figure 15. Heat Exchange Network (HEN) structure

The problem can be formulated as follows:

(P13):

$\operatorname{Min} Z=\sum_{i} C P_{i}+F C P_{h}\left(T_{1}-T_{\text {out }, h}\right) C_{\text {cu }}+F C P_{c}\left(T_{\text {out }, c}-T_{2}\right) C_{\text {hu }}$

s.t.

$F C P_{h}\left(T_{i n, h}-T_{1}\right)=A_{1} U_{1} \frac{\left(T_{i n, h}-T_{2}\right)+\left(T_{1}-T_{i n, c}\right)}{2}$

$F C P_{h}\left(T_{i n, h}-T_{1}\right)=A_{2} U_{2} \frac{\left(-T_{i n, c w}+T_{o u t, h}\right)+\left(T_{1}-T_{\text {out }, c w}\right)}{2}$

$F C P_{c}\left(T_{\text {out }, c}-T_{2}\right)=A_{3} U_{3} \frac{\left(T_{s}-T_{2}\right)+\left(T_{s}-T_{\text {out }, c}\right)}{2}$

$F C P_{h}\left(T_{i n, h}-T_{1}\right)=F C P_{c}\left(T_{2}-T_{i n, c}\right)$

$\left[\begin{array}{c}Y_{1 i} \\ C P_{i}=2750 A_{i}^{0.6}+3000 \\ 0 \leq A_{i} \leq 10\end{array}\right] \underline{\mathrm{v}}\left[\begin{array}{c}Y_{2 i} \\ C P_{i}=1500 A_{i}^{0.6}+15000 \\ 10 \leq A_{i} \leq 25\end{array}\right] \underline{\mathrm{v}}\left[\begin{array}{c}Y_{3 i} \\ C P_{i}=600 A_{i}^{0.6}+46500 \\ 25 \leq A_{i} \leq 50\end{array}\right] \mathrm{i}=1,2,3$

$T_{1}^{l o} \leq T_{1} \leq T_{1}^{u p}$

$T_{2}^{l o} \leq T_{2} \leq T_{2}^{\text {up }}$ 
$Y_{i j} \in\{\text { True, False }\}_{i=1,2,3} j=1,2,3$

$T_{i} \in R^{1} \quad i=1,2$

$A_{i} \in R^{1} \quad i=1,2,3$

where,

$F C P_{i}$ : Heat Capacity of stream i , where i $\in\{$ h,c $\}$

$T_{i n, i}$ : Inlet temperature conditions of the stream $\mathrm{i}$, where $\mathrm{i} \in\{\mathrm{h}, \mathrm{c}\}$

$T_{\text {out }, i}$ : Outlet temperature conditions of the stream $i$, where $i \in\{h, c\}$

$U_{i}$ : Overall Heat Transfer Coefficient for exchanger $E_{i}$, where $i \in\{1,2,3\}$

$C_{h u}$ : Cost of hot utility

$C_{c w}:$ Cost of cooling water

$T_{1,2}$ : Intermediate temperatures

$A_{i}$ : Area of heat exchanger $\mathrm{E}_{\mathrm{i}}$, where $\mathrm{i} \in\{1,2,3\}$

The parameters for this problem are shown in Table 6 .

Table 6: Data for Heat Exchange Network example

\begin{tabular}{|c|cc}
\hline \multicolumn{1}{c}{ Heat Exchanger } & Area $\left.\mathbf{( m}^{\mathbf{2}}\right)$ & Investment Cost (\$/yr) \\
\hline \multirow{3}{*}{1,2 and 3} & $0 \leq \mathrm{A} \leq 10$ & $2750 \mathrm{~A}^{0.6}+3000$ \\
& $10 \leq \mathrm{A} \leq 25$ & $1500 \mathrm{~A}^{0.6}+15000$ \\
& $25 \leq \mathrm{A} \leq 50$ & $600 \mathrm{~A}^{0.6}+46500$ \\
\hline
\end{tabular}

\begin{tabular}{cc}
\hline Heat Exchanger & Overall Heat Transfer \\
\hline 1 & 1.5 \\
2 & 0.5 \\
3 & 1 \\
\hline
\end{tabular}

\begin{tabular}{ccccc}
\hline Stream & $\mathbf{F C P}(\mathbf{k W} / \mathbf{K})$ & $\mathbf{T}_{\mathbf{i n}}(\mathbf{K})$ & $\mathbf{T}_{\mathbf{\text { out }}}(\mathbf{K})$ & $\mathbf{C o s t} \mathbf{( \$ \mathbf { k W } \text { } \mathbf { ~ r } )}$ \\
\hline Hot & 10.0 & 500 & 340 & \\
Cold & 7.5 & 350 & 560 & \\
Cooling Water & & 300 & 320 & 20 \\
Steam & & 600 & 600 & 80 \\
\hline
\end{tabular}

Table 7 shows the solution, size of the problem and tightness of the relaxation ${ }^{[34]}$. Note that the relaxation in this case represents the lower bound of the global minimum $(144,385)$. In this case, the HR after basic steps yield an increase of 3.5\% in the lower bound, and interestingly requires less time than the HR without basic steps. 
Table 7: Results for HEN problem.

\begin{tabular}{lcc} 
& $(\mathrm{HR})$ & $(\mathrm{HR})$ after Basic Steps \\
\hline Optimal solution & 114,385 & 114,385 \\
Relaxation & $\mathbf{9 1 , 6 7 1}$ & $\mathbf{9 4 , 9 2 6}$ \\
Number of constraints & 87 & 206 \\
Number of variables & 52 & 106 \\
Number of binaries & 9 & 9 \\
Number of nodes & 10 & 1 \\
Solution time (s) & 9.0 & 5.0 \\
\hline $1 . \quad$ Selection of disjunctions to apply the basic steps is discussed in source &
\end{tabular}

\section{Concluding Remarks}

This paper has given a general overview of Generalized Disjunctive Programming (GDP), a systematic modeling framework that represents discrete-continuous optimization problems in terms of algebraic equations and high level symbolic logic expressions. Several examples in process systems engineering including optimization of flowsheet superstructures, design of distillation columns, design of batch processes and to scheduling problems have been presented to illustrate how GDP greatly facilitates the modeling of these problems. Furthermore, big-M and hull relaxation reformulations have been presented as two major mixed-integer algebraic models that can be systematically derived from a GDP model. The former is smaller in size, but the latter exhibits stronger continuous relaxations, and hence stronger lower bounds, which may potentially translate into a more efficient solution times, although this is not always the case due to the increased size of the reformulation.

Furthermore, the concept of basic steps for reformulation in GDP models has been presented in order to further improve the continuous relaxations of these problems. In the limit where the disjunctions are converted into Disjunctive Normal Form through these basic steps, a perfect mixed-integer formulation can be obtained that corresponds to the convex hull, meaning that the mixed-integer problem can be solved as a continuous optimization problem. The application of basic steps following some basic rules have been illustrated with a number of computational results from several examples in the area of process systems engineering showing that the predicted lower bounds can be significantly improved, including the case of global optimization problems. Finally, it is hoped that this paper will help to clarify the scope of GDP as a fundamental theoretical framework for modeling discrete-continuous optimization problems.

\section{Acknowledgment}

The authors would like to acknowledge financial support from the National Science Foundation under Grant OCI-0750826. Ignacio Grossmann would also like to acknowledge the work of his former Ph.D. students, Ramesh Raman, Metin Turkay, Sangbum Lee, Nicolas Sawaya, Juan Pablo Ruiz and Hector Yeomans, who have made possible the exciting developments in the area of Generalized Disjunctive Programming. Ignacio Grossmann also would like to acknowledge Professor Egon Balas from Carnegie Mellon for his inspiring and pioneering work in the area of disjunctive programming. 


\section{References}

[1] Balas E., "Disjunctive Programming: Properties of the Convex Hull of Feasible Points", MSRR \#348, Carnegie Mellon University, Pittsburgh, PA, July 1974.

[2] Balas E., "Disjunctive Programming and a Hierarchy of Relaxations for Discrete Continuous Optimization Problems”, SIAM J. Alg. Disc. Meth., Vol. 6, No. 3, 1985.

[3] Beaumont N. "An Algorithm for Disjunctive Programs", European Journal of Operations Research, 48, 362-371, 1991.

[4] Biegler L.T., Grossmann I.E., Westerberg A.W., "Systematic methods of chemical process design”, Prentice Hall, 1997

[5] Birewar D.B. and Grossmann I.E., "Simultaneous synthesis, sizing, and scheduling of multiproduct batch plants”, Industrial and Engineering Chemistry Research, 29 (11), pp 22422251, 1990.

[6] Castro P., Grossmann I.E. “Generalized Disjunctive Programming as a Systematic Modeling Framework to Derive Scheduling Formulations”, Industrial and Engineering Chemistry Research, 51, 5781-5792, 2012.

[7] Cavalier T.M., Pardalos P.M. and Soyster A.L. "Modeling and Integer Programming Techniques Applied to Propositional Calculus”, Computers Opns Res. Vol. 17, No. 6. pp. 561570, 1990.

[8] Clocksin W.F. and Mellish C.S., "Programming in Prolog”, New York: Springer Verlag.

[9] Ierapetritou M.G., Floudas C.A., "Effective continuous-time formulation for short-term scheduling. 1. Multipurpose batch processes.”, Industrial and Engineering Chemistry Research 37, 4341, 1998.

[10] Furman K.C. and Sahinidis N.V., “A Critical Review and Annotated Bibliography for Heat Exchanger Network Synthesis in the 20th Century”, Industrial and Engineering Chemistry Research, 41 (10), 2335-2370, 2002.

[11] Grossmann I.E. and Kravanja Z., "Mixed-integer nonlinear programming techniques for process systems engineering”, Computers \& Chemical Engineering, 19, Supplement 1, 189-204, 1995.

[12] Grossmann I.E., Caballero J.A. and Yeomans H., "Advances in Mathematical Programming for Automated Design, Integration and Operation of Chemical Processes", Korean J. Chem. Eng., 16, 407-426, 1999.

[13] Grossmann, I.E. "Review of nonlinear mixed-integer and disjunctive programming techniques”, Optimization and Engineering, 3, 227-252, 2002

[14] Grossmann I.E. and Lee S., "Generalized Convex Disjunctive Programming: Nonlinear Convex Hull Relaxation”, Computational Optimization and Applications, 26, 83-100, 2003.

[15] Grossmann I.E., Aguirre P.A., Barttfeld M, “Optimal synthesis of complex distillation columns using rigorous models”, Computers and Chemical Engineering 29, 1203-1215, 2005. 
[16] Grossmann I.E. and Ruiz J.P., "Generalized Disjunctive Programming: A Framework for Formulation and Alternative Algorithms for MINLP Optimization," IMA Volume 154, Mixed Integer Nonlinear Programming (eds., Jon Lee and Sven Leyffer), 2011.

[17] Grossmann I.E., "Advances in mathematical programming models for Enterprise-wide Optimization”, Computers \& Chemical Engineering, Available online 5 July, 2012

[18] Hart W.E., Laird C., Watson J.P., Woodruff D.L., "Pyomo - Optimization Modeling in Python”, Springer, 2012.

[19] Hooker J.N. and Osorio M.A., "Mixed logical.linear programming", Discrete Applied Mathematics, 96-97, pp.395-442, 1999.

[20] Hooker J.N., "Logic-Based Methods for Optimization: Combining Optimization and Constraint Satisfaction”, Wiley, 2000.

[21] Kallrath J., "Mixed Integer Optimization in the Chemical Process Industry: Experience, Potential and Future”, Trans. I .Chem E., 78, Part A, pp.809-822, 2000.

[22] Kallrath J. Editor, "Algebraic Modeling Systems: Modeling and Solving Real World Optimization Problems”, Springer, 2012.

[23] Karuppiah R. and Grossmann I.E., "Global Optimization for the Synthesis of Integrated Water Systems in Chemical Processes”, Computers \& Chemical Engineering, 30, issue 4, 650673, 2006.

[24] Kondili E., Pantelides C.C. and Sargent R.W.H., "A general algorithm for short-term scheduling of batch operations - I. MILP formulation”, Computers and Chemical Engineering 17, 211-227, 1993.

[25] Lee S. and Grossmann I.E., "New Algorithms for Nonlinear Generalized Disjunctive Programming”, Computers and Chemical Engineering, 24, 2125-2141, 2000.

[26] Lee S. and Grossmann I.E., "Logic-based Modeling and Solution of Nonlinear Discrete/Continuous Optimization Problems”, Annals of Operations Research, 139, Number 1, 267-288, 2005

[27] McCormick G.P., "Computability of global solutions to factorable nonconvex programs. Part I. Convex underestimating problems”, Mathematical Programming, 10, 146-175, 1976.

[28] Méndez C.A., Henning G.P., Cerdá,J., “An MILP continuous time approach to short-term scheduling of resource-constrained multistage flowshop batch facilities.”, Computers and Chemical Engineering 25, 701, 2001.

[29] Méndez C.A., Cerdá J., Grossmann I.E., Harjunkoski I., Fahl M., “State-of-the-art Review of Optimization Methods for Short-Term Scheduling of Batch Processes", Computers and Chemical Engineering 30, 913, 2006.

[30] Nemhauser G.L. and Wolsey L.A., "Integer and Combinatorial Optimization, WileyInterscience”, 1988.

[31] Raman R. and Grossmann I.E., "Relation between MILP Modeling and Logical Inference for Chemical Process Synthesis”, Computers and Chemical Engineering, Vol. 15, No. 2, 73-84, 1991. 
[32] Raman R. and Grossmann I.E., "Modelling and Computational Techniques for Logic-Based Integer Programming”, Computers and Chemical Engineering, 18, 563, 1994.

[33] Ruiz J., Thesis: "Global Optimization of Nonconvex Generalized Disjunctive Programming”, Carnegie Mellon University, 2006.

[34] Ruiz J.P. and Grossmann I.E., "Strengthening of lower bounds in the global optimization of Bilinear and Concave Generalized Disjunctive Programs”, Computers and Chemical Engineering, 34, 914-930, 2010.

[35] Ruiz J.P. and Grossmann I.E., “A hierarchy of relaxations for nonlinear convex generalized disjunctive programming”, European Journal of Operational Research, 218, issue 1, 38-47, 2012.

[36] Sargent R.W.H. and Gaminibandara K., “Optimal Design of Plate Distillation Columns”, Optimization in Action; Dixon, L. C. W., Ed; Academic Press: New York, 1976

[37] Sawaya N., Thesis: "Reformulations, Relaxations and Cutting Planes for Generalized Disjunctive Programming”, Carnegie Mellon University, 2006.

[38] Sawaya N. and Grossmann I.E., "A hierarchy of relaxations for linear generalized disjunctive programming”, European Journal of Operational Research, 216 70-82, 2012.

[39] Trespalacios F. and Grissmann I.E., “An algorithmic approach for improving convex generalized disjunctive programming models through the application of basic steps”, in process

[40] Türkay M. and Grossmann I.E., "A Logic Based Outer-Approximation Algorithm for MINLP Optimization of Process Flowsheets", Computers and Chemical Engineering, 20, 959978, 1996.

[41] Vecchietti A., Grossmann I.E., "Modeling issues and implementation of language for disjunctive programming”, Computers and Chemical Engineering 24, 2143-2155, 2000.

[42] Vecchietti A., Lee S., Grossmann I.E. "Modeling of discrete/continuous optimization problems: characterization and formulation of disjunctions and their relaxations”. Computers and Chemical Engineering 27, 433-448, 2003.

[43] Viswanathan J. and Grossmann I.E., "An Alternative MINLP Model for Finding the Number of Trays Required for a Specified Separation Objective”, Computer and Chemical Engineering, 17 (9), 949, 1993.

[44] Williams H.P., “Model Building in Mathematical Programming”, Wiley, 1985.

[45] Yeomans H. and Grossmann I. E., "Disjunctive programming models for the optimal design of distillation columns and separation sequences”, Industrial and Engineering Chemical Research, 39(6), 1637, 2000. 


\section{Appendices}

\section{Appendix 1. Embedded disjunctions and inclusive OR Formulations}

(GDP) represents the general form of Generalized Disjunctive Programming. However, there are logic models that are formulated more intuitively in different forms than (GDP). Two particular cases are embedded disjunctions ${ }^{[41]}$ and inclusive OR arguments.

\section{Embedded Disjunctions}

Embedded disjunctions may arise in PSE problems (see for example (P6) for discontinuous cost functions). An embedded disjunction is illustrated in (P14.1):

\section{(P14.1):}

$\left[\begin{array}{c}Y_{1} \\ r_{1}(x) \leq 0 \\ {\left[\begin{array}{c}Y_{11} \\ r_{11}(x) \leq 0\end{array}\right] \underline{\mathrm{v}}\left[\begin{array}{c}Y_{12} \\ r_{12}(x) \leq 0\end{array}\right]}\end{array}\right] \underline{\mathrm{v}}\left[\begin{array}{c}Y_{2} \\ r_{2}(x) \leq 0\end{array}\right]$

which implies that if $Y_{1}=T R U E$, then $r_{1}(x) \leq 0$ must be satisfied, and there is an additional disjunction $Y_{11}$ XOR $Y_{21}$. If $Y_{2}=T R U E$ then $r_{2}(x) \leq 0$ must be satisfied. To achieve (GDP) form, (P14.1) can be transformed as follows:

\section{(P14.2):}

$\left[\begin{array}{c}Y_{1} \\ r_{1}(x) \leq 0\end{array}\right] \underline{\mathrm{v}}\left[\begin{array}{c}Y_{2} \\ r_{2}(x) \leq 0\end{array}\right]$

$\left[\begin{array}{c}Y_{11} \\ r_{11}(x) \leq 0\end{array}\right] \underline{\vee}\left[\begin{array}{c}Y_{12} \\ r_{12}(x) \leq 0\end{array}\right] \underline{\vee}\left[Y_{13}\right]$

$Y_{1} \Leftrightarrow Y_{11} \underline{\vee} Y_{12}$

It is easy to see that (P14.1) and (P14.2) are logically equivalent.

Inclusive OR

Another common logic proposition appears when the disjunctions are not necessarily exclusive (i.e. OR vs. XOR; ₹ vs. ํ). (P15.1) illustrates an inclusive OR disjunction:

\section{(P15.1):}

$\left[\begin{array}{c}Y_{1} \\ r_{1}(x) \leq 0\end{array}\right] \vee\left[\begin{array}{c}Y_{2} \\ r_{2}(x) \leq 0\end{array}\right]$

Note that $Y_{1}$ and $Y_{2}$ cannot both be false (i.e. $Y_{1}$ can be TRUE and $Y_{2}$ FALSE; or $Y_{1}$ FALSE and $Y_{2}$ TRUE, or $Y_{1}$ TRUE and $Y_{2}$ TRUE). This means that it is possible to enforce both $r_{1}(x) \leq 0$ and $r_{2}(x) \leq 0$. To achieve (GDP) form, (P15.1) can be formulated as follows: 
(P15.2):

$\left[\begin{array}{c}Y_{1} \\ r_{1}(x) \leq 0\end{array}\right] \underline{v}\left[\neg Y_{1}\right]$

$\left[\begin{array}{c}Y_{2} \\ r_{2}(x) \leq 0\end{array}\right] \underline{\vee}\left[\neg Y_{2}\right]$

$Y_{1} \vee Y_{2}$

(P15.1) and (P15.2) are logically equivalent.

\section{Appendix 2. Review of GDP methods}

Two solution methods have been proposed for direct solution convex nonlinear GDP, namely, the Branch and Bound method by Lee and Grossmann ${ }^{[25]}$, which builds on the concept of disjunctive Branch and Bound method by Beaumont ${ }^{[3]}$, and the Logic Based OuterApproximation method by Turkay and Grossmann ${ }^{[40]}$.

The basic idea in the $\mathbf{B} \& \mathbf{B}$ method is to directly branch on the constraints corresponding to particular terms in the disjunctions, while considering the convex hull of the remaining disjunctions. Although the tightness of the relaxation at each node is comparable with the one obtained when solving the HR reformulation with a MINLP solver, the size of the problems solved are smaller and the numerical robustness is improved.

For the case of Logic Based Outer-Approximation methods, similar to the case of OA for MINLP, the main idea is to solve iteratively a master problem given by a linear GDP, which will provide a lower bound of the solution, and an NLP subproblem that will yield an upper bound. As described in Turkay and Grossmann ${ }^{[40]}$, for fixed values of the Boolean Variables, $Y_{k \hat{i}}=$ true , $Y_{k i}=$ false with $\hat{i} \neq i$, the corresponding NLP subproblem (SNLP) is as follows:

Min $Z=f(x)$

s.t. $g(x) \leq 0$

$r_{k i}(x) \leq 0$ for $Y_{k i}=$ true $k \in K, i \in D_{k}$

(SNLP)

$x^{l o} \leq x \leq x^{u p}$

$x \in R^{n}, c_{k} \in R^{1}, Y_{k i} \in\{$ True, False $\}$

It is important to note that only the constraints that belong to the active terms in the disjunction (i.e. associated Boolean variable $Y_{i k}=$ true) are imposed. Constraints involved in the inactive disjunctive terms are disregarded. This leads to a substantial reduction in the size of the problem compared to the direct application of the traditional OA method on the MINLP reformulation (as described in section 2). Assuming that $\mathrm{L}$ subproblems are solved in which sets of linearizations $\ell=1,2 \ldots . . L$ are generated for subsets of disjunction terms $L_{k i}=\left\{\ell \mid Y_{k i}^{\ell}=\right.$ True $\}$, one can define the following disjunctive OA master problem (MLGDP):

$\operatorname{Min} Z \stackrel{\text { Error }}{=} \alpha$

Error! Bookmark not defined. 
s.t.

$\left.\begin{array}{l}\alpha \geq f\left(x^{\ell}\right)+\nabla f\left(x^{\ell}\right)^{T}\left(x-x^{\ell}\right) \\ g\left(x^{\ell}\right)+\nabla g\left(x^{\ell}\right)^{T}\left(x-x^{\ell}\right) \leq 0\end{array}\right\} \ell=1,2 \ldots . ., L$

$\underset{i \in D_{k}}{\vee}\left[\begin{array}{l}Y_{k i} \\ r_{k i}\left(x^{\ell}\right)+\nabla r_{k i}\left(x^{\ell}\right)\left(x-x^{\ell}\right) \leq 0 \quad \ell \in L_{k i}\end{array}\right] \quad k \in K \quad$ (MLGDP)

$\Omega(Y)=$ True

$x^{l o} \leq x \leq x^{u p}$

$\alpha \in R^{1}, x \in R^{n}, c_{k} \in R^{1}, Y_{k i} \in\{$ True,False $\}$

It should be noted that before applying the above master problem it is necessary to solve various subproblems (SNLP) for different values of the Boolean Variables $Y_{i k}$ so as to produce one linear approximation of each of the terms $i \in D_{k}$ in the disjunctions $k \in K$. As shown by Turkay and Grossmann $^{[40]}$ selecting the smallest number of subproblems amounts to solving a set covering problem, which is of small size and easy to solve. It is important to note that the number of subproblems solved in the initialization is often small since the combinatorial explosion that one might expect is in general limited by the propositional logic. Moreover, terms in the disjunctions that contain only linear functions need not be considered for generating the subproblems. This frequently arises in process networks since they are often modeled by using two terms disjunctions where one of the terms is always linear (see remark below). Also, it should be noted that the master problem can be reformulated as an MILP by using the big-M or Convex Hull reformulation, or else solved directly with a disjunctive branch and bound method. 